\title{
Generations of Knowledge Management
}

\author{
J o seph M. F ir estone, Ph.D. \\ Execut ive Infor mation Systems, Inc.
}

And

\author{
Mark W. McEIroy \\ Macro innovation Associates, LLC
}

J ul y 2002

\section{Three Views of Change in Knowledge Management}

Knowledge Management, new as it is, is changing. There are at least three accounts of how it is changing and about how we should view "The New Knowledge Management" (TNKM). One account, by Mark Koenig (2002), sees $\mathrm{KM}$ as a field that was originally driven by information technology, the Internet, best practices, and later lessons learned, and most importantly knowledge sharing. This theory sees a second stage of $\mathrm{KM}$ as about human factors, organizational learning, and knowledge creation viewed as the conversions among tacit and explicit knowledge. The third stage of KM is the stage of the arrangement and management of content through taxonomy construction and use, and like the first is also heavily biased towards information technology.

The second view of change, by David Snowden (2002), is a bit more subtle than the first. According to this theory, the first age of knowledge management is one in which the word knowledge itself was not at first "problematic," and in which the focus was on distributing information to decision makers for timely use in decisions. The second age replaced the information technology focus with one on tacit/explicit knowledge conversion inspired by Nonaka's SECI model. It is just ending. Snowden contends that the third age will be one in which: knowledge is viewed paradoxically as a thing and a flow; context, narrative and content management will be central to our view of KM; Further, he believes that there will be an understanding of organizations as engaged in sensemaking through utilization of complex adaptive systems (CAS) phenomena 
constrained by human acts of free will attempting to order them; and finally, the use of the insights and practices of scientific management will be restricted to appropriate contexts, while "insights and learnings" from theories of chaos and complexity will supplement them in contexts where these new insights are relevant.

The third view of change, first presented by Mark W. McElroy (1999) based on work hosted by the Knowledge Management Consortium International $(\mathrm{KMCl})$ and continuing partly under its auspices since then, views first generation $\mathrm{KM}$, also called "supply-side $\mathrm{KM}$," as primarily about integrating ("supplying") previously created knowledge through knowledge distribution, sharing, and other integrative activities. It is typically associated with two well-known phrases that serve as the mantras for advocates of the 'knowledge sharing' side of KM: (1) It's all about capturing, codifying, and sharing valuable knowledge, and (2) It's all about getting the right information to the right people at the right time. The third view sees second generation $\mathrm{KM}$ as first appearing in the mid-90s and as being focused not only on "supply-side" knowledge processing such as knowledge sharing, but also on "demand-side" knowledge processing, or "knowledge-making" in response problem-induced demands. This combined focus on knowledge integration and knowledge production is the defining characteristic of second generation KM (SGKM), or alternatively, The New Knowledge Management (TNKM). But an important aspect of it is also the recognition that organizations are permeated with complex adaptive systems phenomena, and that knowledge management in them is about using $\mathrm{KM}$ to enable or reinforce self-organization in knowledge processing for the purpose of achieving sustainable innovation in support of organizational adaptation.

So in light of these contrasting views the questions arise: which of the three views is correct? Are there two generations, stages or ages of KM? Is a third age about to begin? Or are there already three? Are the changes best seen as occurring along the information technology dimension? Or along linguistic dimensions such as taxonomy construction, context, and narrative? Or in terms of whether we view organizations as mechanisms, or CASs, or CASs modified by human Promethean interventions? Or just in terms of the popularity of different intervention types from one period to another? Or is change in KM best viewed as occurring in terms of the shifting focus of management on the scope of knowledge processing as identified by McElroy? We will answer these questions after we have examined each of the three views in more detail. 


\section{The Three Stages of Knowledge Management}

In Mark Koenig's view (2002, p. 20):

The initial stage of KM was driven primarily by information technology. That stage has been described . . . as "by the Internet out of intellectual capital."

By this, Koenig means that the development of the Internet and the use of its technology to implement intranets provided the enterprise with an unprecedented tool for knowledge sharing and transfer, and thus for getting value out of their previously developed intellectual capital. Knowledge management was the name introduced to describe the management activity concerned with implementing such solutions, in order to gain competitive advantage and to increase productivity and effectiveness. Further, this activity could be rationalized by its proponents (including large consulting organizations selling their own newly developed expertise in implementing such solutions) in terms of increasing the value of an enterprise's intellectual capital (IC). The notion of IC had appeared a few years earlier to account for the increasing disparity between the market value of real world enterprises and their book value as computed using measurable financial indicators and conventional formulae for establishing company valuations. In addition to the above, the first stage of KM was also characterized by a focus on "best practices," later revised to a focus on "lessons learned." But it is not clear from Koenig's account what "best practices" and "lessons learned" in KM have to do with the IT focus supposedly dominant in stage one.

So for Koenig, the first stage of KM was about applying technology to accomplish knowledge sharing and coordination across the enterprise. The second stage, on the other hand was primarily a recognition that KM was not only about applying technology, but was also about human and cultural factors as essential in implementing KM applications, if failures were to be avoided. Koenig calls attention to the work of Senge (1990) on organizational learning, and Nonaka and Takeuchi (1995) on the SECI model and its applications, as both being essential to phase two. Koenig also calls attention to the focus of these works on organizational learning and knowledge creation, but not on systems thinking, which was an important element in Senge's treatment and in the rising popularity of stage two activities. Koenig also mentions the importance of communities of practice in stage two, but neglects to make the connection between the communities of practice emphasis and the thinking of Senge and Nonaka 
and Takeuchi, or for that matter between communities of practice and knowledge creation and innovation.

Koenig thinks that the third stage of $\mathrm{KM}(2002$, p. 21) "is the awareness of the importance of content-and, in particular, an awareness of the importance of the retrievability and therefore of the arrangement, description and structure of that content." In particular, the third stage is about finding relevant content, and about taxonomy development and content management to facilitate this goal.

\section{Difficulties with the Three Stages View}

There are a number of difficulties in Koenig's account of the development of knowledge management. First, the dates in his account of development are unclear. His theory is one of the onset of new KM stages which then exist along with the old. If such a stage theory of $\mathrm{KM}$ development is to be applied, we need either clear dates to distinguish the the beginning of each successive stage, or a clear period of transition in which the previous stage is gradually supplemented by the features of the new stage. Without these criteria it is very hard to characterize a particular period as stage one rather than stage two and to confirm that analysis. Specifically, Koenig claims that stage one precedes stage two and that stage one is about applying IT, while stage two is about the human element in KM. Yet Senge's book, identified as a stage two milestone, was written in 1990 much before the trend toward intranets and knowledge sharing applications, supposedly characteristic of stage one, gained momentum. Karl Wiig's work (1989) introducing $\mathrm{KM}$ as a field was also written much before the widespread adoption of Internet-based technology in enterprises, and even before Senge's book. And then Wiig wrote three books in 1993-1995, looking at KM comprehensively. Even Nonaka and Takeuchi dates from 1995, a date that surely preceded the heyday of so-called stage one $\mathrm{KM}$ with its emphasis on intranet-based knowledge sharing and coordination applications.

The same difficulty applies to the supposed transition from stage two to stage three KM. If we are to believe Koenig, it was not until the year 2001 that content management and taxonomy development became important for KM. However, The 1998 combined KM World/AllM conference held in Chicago had a major content management element. In fact, attendees were frequently heard by these authors to complain that content management was dominating the conference and that such applications were not KM applications, and that claims to the contrary were merely vendor speak hiding the fact that vendors had very few real KM applications to offer.

Following that event, moreover, the conference circuit began to see an explosion of content management and portal conferences. These were 
often closely associated with so-called KM applications. Both types of conferences included sessions on tools for taxonomy development and for increasingly efficient retrieval of information through application of search technology.

While there is no question that the interest in content management and taxonomy development continues to increase, there is no reason to claim that this trend is either later than the second stage or even that such activities are new and go beyond the concerns of the second stage. After all, the concern with taxonomy and content management is about more efficiently retrieving knowledge or information that already exists. Thus, its core motivating concern is not different from Koenig's stage one. That is, taxonomy development and content management are primarily about coordinating and sharing already existing knowledge, and only secondarily about aiding knowledge making. So there is a good argument for asserting that Koenig's stage 3 is really just an extension of his stage one.

Second, another difficulty with Koenig's account is the ad hoc character of its classification of the three stages of KM, apparently based on anecdote and personal observation. There is no underlying conceptual framework organizing the analysis of change. KM supposedly begins as an IT field. Then suddenly, under the influence of Senge and Nonaka, it begins to incorporate the human element and this element is apparently either discontinuous with what has gone before, or is a response to the perceived failure of the IT applications implemented in the first age. It is presented as if it is a mere adaptation to a problem, the problem of getting organizations to accept IT interventions defined as KM projects. Further, a similar ad hoc adaptation is viewed as triggering the move from stage two to stage 3 . That is, Koenig seems to think that we have a new stage of KM because people realize that they can't either share or create knowledge without good webbased navigation to help them "find it."

The third difficulty with Koenig's view is that its lack of a conceptual framework provides only limited guidance for the further development of $\mathrm{KM}$. He points to the fact that his view suggests that librarians may have an important part to play in taxonomy development as a positive reason for taking it seriously. But what does his analysis imply about knowledge management activities or policies more generally, or about knowledge production, organizational learning, sustainable innovation, intellectual capital, KM metrics, methodologies, and IT requirements for KM applications? That is, once we understand his account, what can we do with it? The answer is, very little. Unless one is interested in content management and taxonomy development, there is little of interest in it, or of general significance for the further development of KM. Thus, it provides both an inadequate analysis of the past and fails to provide a road map for the future. 
In brief, we believe that Koenig's approach to the analysis of stages of KM is much too ad hoc in character, focusing on tools and techniques and not on the broad purposes of $\mathrm{KM}$. As a result, his typology only records shifting fashions, not fundamental shifts in disciplinary concerns. Thus, he cannot recognize that both IT applications in support of KM and taxonomy/content management concerns are not about central issues of knowledge management orientation, but rather are about techniques and tools for supporting such KM orientations. The development of these techniques and tools, however, is driven by the basic orientations and purposes themselves.

So, current content management, taxonomy, and portal application concerns are about supporting knowledge coordination and transfer applications. They are not yet about supporting knowledge making, production, and creation. This is true because first generation $\mathrm{KM}$ is still dominant, while second generation KM (SGKM) (originating as a coherent orientation toward the subject somewhere in the period 1995-1999) is still in the process of taking hold. This point will become clearer as we get into the discussion of the Snowden and McElroy views on change in KM.

\section{The Two Ages of Knowledge Management (With a Third Yet to Come)}

According to Snowden (2002, p. 100) the first age of KM, prior to 1995, was about:

"the appropriate structuring and flow of information to decision makers and the computerisation of major business applications leading to a technology enabled revolution dominated by the perceived efficiencies of process engineering."

He calls this age "information for decision support." And his characterization of it, as the above quote implies, does not distinguish it from Business Process Re-engineering (BPR). In fact, for Snowden, KM in the first age seems to be a species of BPR, which proceeded without recognition of knowledge gained through experience or person-to-person processes of knowledge transfer. Since it ignored these aspects of knowledge, Snowden thinks that the word knowledge itself became problematic in KM by the end of the first age.

Snowden further contends (2002, p. 101) that the second age of knowledge management began in 1995 with "the popularisation of the SECI Model" after the publication of Nonaka and Takeuchi's The Knowledge Creating Company (1995). But he also says that "to all intents and purposes knowledge management started circa 1995" (ibid.) with the publication of this book. That statement combined with his characterization of the first age as one of information for decision support raises the 
question of why there is a first age in his change framework at all, or more properly why his second age of knowledge management is not his first age.

The second age of KM in Snowden's view is characterized by a focus on the four SECl model's processes describing the conversion of knowledge from Explicit to Tacit (Socialization), Tacit to Explicit (Externalization), Explicit to Explicit (Combination), and Tacit to Tacit (Internalization). He goes on to comment about the misunderstanding of Polanyi's $(1958,1966)$ views on the nature of the relationship between tacit and explicit knowledge that is prevalent in the second age, specifically that it was "dualistic, rather than dialectical" (ibid.), in contrast with both Polanyi's and Nonaka's understanding of the relationship. But he says little else about the second age, leaving the impression that there was little else to this age of KM.

\section{Difficulties with the Two Ages View}

Before moving on to Snowden's characterization of the coming third age of knowledge management, note that his description of the first two ages of $\mathrm{KM}$ leaves a lot of open questions. First, was there really no more to the first age of KM than "information for decision support"? If so, then why was the term KM used at all? After all, the field of business intelligence provides information for decision support. So do data warehousing and data mining. And so does the still broader category of Decision Support Systems (DSS). So what was the term KM supposed to signify that those other terms do not?

Second, also, if there was no more than information for decision support to the first age, then what were the attempts to distinguish data, information, knowledge and wisdom about? What was the development of Xerox's community of practice for the exchange of knowledge among technicians about? What was knowledge sharing at Buckman laboratories in 1987 and 1992 (Rumizen, 1998) about? Where does Hubert St. Onge's work (See Stewart, 1999) on the relationship of customer capital to the learning organization fit? Or Senge's (1990) work on systems thinking? Or Karl Wiig's early introductions to KM $(1993,1994,1995)$ ?

In brief, Snowden's characterization of the first age of KM as focused on providing information for decision support and implementing BPR schemes suggests much too heavy an emphasis on KM as primarily composed of IT applications to reflect the full reality of the first age. In fact, his failure to take account of the human side of KM during the first age suggests a desire for the same kind of neat distinction we find in Koenig's analysis. In effect, Snowden, like Koenig, seems to want to say that the first age was about technology and the second age was about the role of people in Nonaka's four modes of conversion. 
Third, in describing the second age of KM, Snowden's account is, once again, far too spare in its characterization. No doubt, the Nonaka and Takeuchi book has had an important and substantial impact on KM, but the period since 1995 has seen important work done in many areas not explicitly concerned with knowledge conversion.

These areas include semantic network analysis, the role of complex adaptive systems theory in knowledge management, systems thinking, intellectual capital, value network analysis, organizational learning, communities of practice, content management, knowledge sharing, conceptual frameworks for knowledge processing and knowledge management, knowledge management metrics, enterprise information portals, knowledge management methodology, and innovation, to name some, but far from all, areas in which important work has been done. Finally, as indicated by the title of Nonaka and Takeuchi's book, their concern, and a central concern of second generation KM as we shall see shortly, is about "knowledge creation," or knowledge production. It is only secondarily about knowledge conversion.

Nonaka mistakenly identified knowledge creation wholly with knowledge conversion. In our view, however, knowledge conversion only produces belief or psychological knowledge. In the area of producing organizational knowledge as a cultural product, the role of knowledge conversion is focused on only one sub-process in knowledge production the subprocess of knowledge claim formulation. But it doesn't address knowledge claim evaluation or validation, a critical sub-process in the creation of explicit, shared, culturally-based knowledge.

Snowden's account of the coming third age of knowledge management is developed much more carefully in his work than his account of the other two ages. But it will be much easier to reveal, understand and critique his forecast of a third age if we first provide the third view of change in $\mathrm{KM}$, and along with it some additional comments on both the Snowden and Koenig interpretations of generations. We now turn to that task.

\section{The Two Generations of Knowledge Management}

The third perspective on the evolution of KM distinguishes between two "generations": first and second generation KM (first developed in McElroy, 1999). According to this view, a distinction can be made between "supplyside" knowledge management in which interventions are aimed at knowledge integration, or sharing, and demand-side KM which is focused, instead, on knowledge production, or making. In the first case, the practice of $\mathrm{KM}$ is predicated on the assumption that valuable knowledge already exists; the purpose of $\mathrm{KM}$, then, is to capture, codify, and share it. In the second case, no such assumption is made. Before knowledge can be shared, much less captured and codified, it has to first be produced. Thus, 
supply-side KM focuses on enhancing the "supply" of existing knowledge to workers who need it, whereas demand-side KM seeks to enhance our capacity to satisfy our "demands" for new knowledge.

First generation $\mathrm{KM}$, according to this view, was (and continues to be) supply-side only in its orientation. Second generation practice, however, is both supply- and demand-side oriented. Of crucial importance to this view of $\mathrm{KM}$, then, is the contention that knowledge is not only something we share, but is also something we make. Indeed, we can only share knowledge that exists, and knowledge can exist only after it is created - by people.

A process-oriented (and cyclical) view of knowledge making and sharing or, more generally, production and integration - therefore comes sharply into focus as a consequence of this third perspective. Rather than assume, as first generation thinkers do, that valuable knowledge already exists and that the sole task of $\mathrm{KM}$ is merely to enhance its distribution, second generation thinkers contend, as well, with the problem of knowledge production. It is because of this that second generation KM is more closely aligned with the fields of organizational learning and innovation management than the second age envisioned by Snowden.

Of additional foundational importance to this third view of $\mathrm{KM}$ is the distinction it makes between knowledge processing and knowledge management. This rather fundamental distinction is not made in either the Koenig or Snowden accounts of the evolution of KM. Knowledge processing is precisely the cycle referred to above, through which people in organizations, in response to problems arising in business processes, collectively engage in knowledge production and integration. Knowledge processes, therefore, are social processes through which organizations make and share their knowledge.

Knowledge management, on the other hand, is a management activity that seeks to enhance knowledge processing. Not all organizations support formal knowledge management functions, but all organizations do engage in knowledge processing. The purpose of $\mathrm{KM}$ according to this view is to enhance an organization's ability to perform knowledge processing, and ultimately by improving it to enhance the quality of its business process behavior and its ability to adapt to its environment.

Also central to the generational view of $\mathrm{KM}$ is the position that knowledge processing in human social systems is a self-organizing affair. That is, people in organizations tend to self-organize in emergent, pattern-like ways around the production and integration of knowledge. We can generally describe the shapes of these patterns using terms like problem detection, intrinsically motivated learning, group and community formation, 
communities of inquiry or practice, problem solving, knowledge evaluation and adoption, knowledge sharing, and so forth. That these activities are self-organizing and pattern-like in their appearance is explained with the aid of complexity and complex adaptive systems theories in the generational view of KM. Indeed, SGKM is deeply rooted in the application of complex adaptive systems theory to knowledge processing in human social systems, a perspective it applies backwards and forwards in its characterization of how KM has evolved over the years.

While the generational view of KM does point to two distinct bodies of practice that are supply-side on the one hand and demand-side on the other, it does not suggest that the second one started only after the first one ended. Rather, the two streams of practice are concurrent in use and will probably carry on in this way for some time to come.

Consider, for example, the field of organizational learning (OL) which is arguably focused on nothing if not demand-side knowledge processing. This is a field that clearly started long before KM (the term) came into fashion in the mid-nineties. The same can be said for the fields of Innovation Management (IM), Organizational Development (OD), and even Human Resources Development (HRD), which has always been concerned with learning and the transfer of knowledge in the form of training and other knowledge sharing programs.

What we have, then, according to the generational view of $\mathrm{KM}$ is two distinct bodies of practice that are separate in content but not necessarily in time. That said, the first generation of KM arguably began quite some time ago, even as early as the late nineteenth/early twentieth centuries with the work of Frederick Taylor (1912) whose Scientific Management system explicitly called for the capture, codification, and use of what we today would refer to as "best practices." This was supply-side KM in action, since its intent was to enhance knowledge capture and sharing. Later on in the twentieth century, work related to enhancing knowledge production began to appear. This took many forms, including the evolution of R\&D and its various methodologies and management schemes, innovation management, organizational learning, and institutionalized science, which has always been concerned with the production of new knowledge. From this perspective, supply- and demand-side $\mathrm{KM}$ is more than one hundred years old.

Despite this, the term "KM" is considerably younger in age. Its initial appearance in the mid-nineties was mostly tied to supply-side $\mathrm{KM}$, and it wasn't until 1999 that the formal distinction between that sense of the term and the new, or second, sense was introduced using the supplyside/demand-side language. Indeed, it wasn't until McElroy (1999) and his colleagues at KMCl (Firestone, 1999, 1999a, 1999b, 1999c) began to think 
of differences in KM practice as being somehow related to the separate and distinct notion of social knowledge processing that the combined form of supply- and demand-side KM was viewed as adding up to a new and unique body of practice (SGKM) deserving of special recognition.

So even though we can say that both supply- and demand-side KM were theoretically being practiced in various forms prior to the advent of "KM" as such in the nineties, they were clearly not being practiced, much less compared to one another, in these terms. Nor were they being tied to a formal conceptual distinction between knowledge processing and $\mathrm{KM}$. That dates from the end of the 1990s, following the advent of KM in the mid-nineties in its supply-side form.

Comparing this third view to the other two, we can see important differences. The Koenig view, as we have said, presents an ad hoc classification scheme in which the three "stages" of KM are based more on anecdote and personal observation than on any sort of underlying conceptual framework related to knowledge processing and how it is practiced. The third, generational view, by contrast, relies explicitly on a vision of social knowledge processing, against which all forms of practice in $\mathrm{KM}$ can be seen and understood, both backwards and forwards in time. Indeed, it is SGKM that is associated with a formal articulation of this vision for just this purpose. That articulation is the Knowledge Life Cycle framework, or KLC, as developed and refined by Firestone (See, for example, Firestone, 1999c, Firestone, 2000, 2000a, 2000b, 2001, 2002), McElroy (1999, 1999a, 2000a, 2000b, 2002, 2002a), and other members of the Knowledge Management Consortium International (Cavaleri and Reed, 2000) over the past four years. The KLC framework is illustrated in Figure 1.

Next, because the scope of the KLC is comprehensive in its representation of knowledge processing in human social systems, all forms of $\mathrm{KM}$ practice, both current and future ones, can be related to it. Everything we do in $\mathrm{KM}$ is designed to have impact on one or more elements of the KLC. A generation of KM, therefore, should not, according to SGKM, be defined in terms of a Koenig-like focus on practice types, tools, or trends. Indeed, such a definition risks losing sight of the central purpose of $\mathrm{KM}$ interventions and tools: to improve knowledge processing. The appearance and/or departure of different kinds or styles of interventions need not - and should not - have anything to do with our attempts to make sense of them in terms of what their basic purpose is.

What is of more fundamental relevance to our analysis of the evolution of $\mathrm{KM}$ is what its practitioners are trying to do, not what their tools and methods are. Of course, we must be interested in tools and methods too, but only after we've settled on purpose, and used it to define the basic 
thrust of KM. Different flavors of tools come and go, but the purpose of KM is always to enhance knowledge processing.

SGKM's conceptual framework for knowledge processing gives rise to another important distinction between it and the Koenig view of stages. As stated earlier, the Koenig view seems to begin and end with an appreciation of the importance of taxonomy development and content management. Under this logic, the very next issue to pop up on the KM landscape would give rise to a fourth stage, a fifth one after that, and so forth. This is what happens when we define evolution from the perspective of tools and styles of interventions - each stage becomes too narrow, too tightly bounded, with nowhere else to go, driven by short-term problems and adaptations. Such a specification of stages is ad hoc and lacks depth of insight into what KM is ultimately trying to achieve or have impact on (i.e., to enhance knowledge processing).

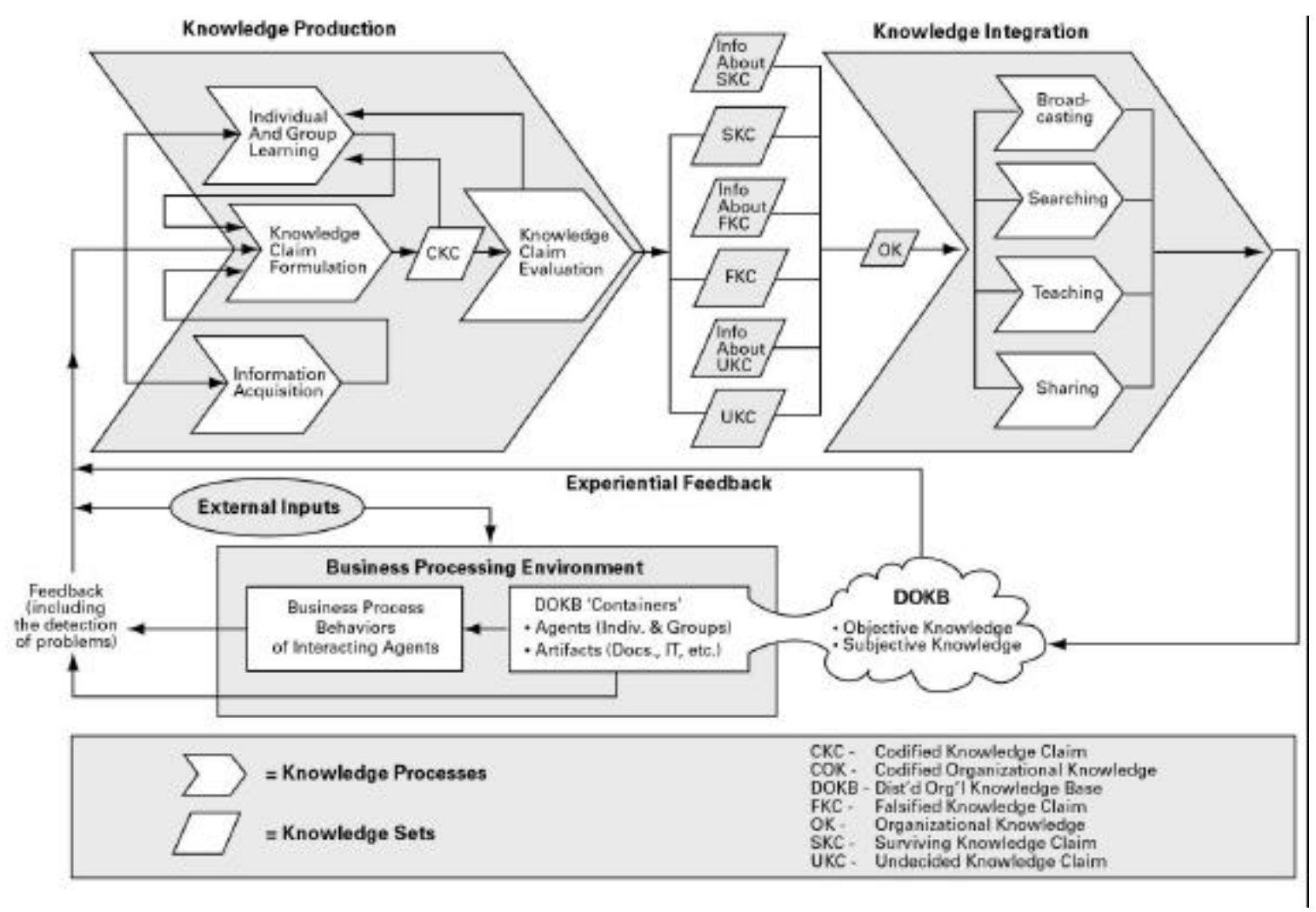

Figure 1 - The Knowledge Life Cycle (KLC) 
By contrast, the third, generational view of $\mathrm{KM}$ is relatively free of bias towards intervention types or styles. According to the generational view, new tools and methods are constantly being created and tested, and there's nothing wrong with that. But what shouldn't (or doesn't) change along the way, except very infrequently, is the intended target of their use. Practitioners of KM either seek to have an impact on knowledge production, knowledge integration, or both. Variations in the tools we use don't change such basic objectives.

In comparing the third, generational view of KM to that of Snowden's, the generational perspective can help us add to our earlier critique of his account of the first two ages of KM. First, his account seems to suffer from the same lack of an underlying conceptual framework used to organize his analysis of change.

In the first age, we are encouraged to envision individuals at work whose momentary needs from time to time require informational support. Then $\mathrm{KM}$ comes to the rescue (or is it IM?), delivers the information, and declares victory. In this view, there is no social system, only individuals. And in this view, there is only the momentary and discrete decision transaction that frames the backdrop for $\mathrm{KM}$, thus failing to distinguish $\mathrm{KM}$, as we pointed out earlier, from data warehousing, business intelligence or DSS. From the perspective of the third, generational view of KM, however, this is an act of knowledge integration (supply-side KM). But this only makes sense in the context of a more comprehensive knowledge processing framework that can help us distinguish knowledge integration from knowledge use. It is a distinction that Snowden does not make, but that is fundamental to first generation, supply-side KM.

In Snowden's second age we are suddenly thrust into the realm of knowledge conversion using Nonaka's SECI model as a reference. This is indeed an improvement over his first age, since his account of the second age characterization at least employs a conceptual framework - some vision of knowledge processing, which arguably goes on between individuals and other individuals, and between groups and individuals and other groups. Nonetheless, we are still left with a fairly narrow frame of reference and a failure to place the Nonaka model into a broader context of knowledge processing or a framework of KM concepts and practice.

This narrow frame of reference, of course, is determined by the scope of the SECI model that Snowden has selected to conceptualize the second age. In the SECI model, knowledge processing is reduced to four kinds of transactions - the "knowledge conversion" transactions reviewed earlier. In using this model as the basis of his second age, Snowden reduces all of KM practice between the years of 1995 and today to a concerted effort on the part of KM professionals to get these four transactions to work better. 
Never mind the much broader scope of KM activity observable since 1995 and discussed earlier. Never mind that he takes us from "information delivery" in the first age to "knowledge conversion" in the second, without explaining the difference between information and knowledge in the transition, or why the SECl model is only about knowledge and not information. And never mind, for that matter, that the SECI model, since it too does not address this question, could just as easily be seen as a way of converting "misinformation" or "falsified knowledge" from one party to another. Or that it could be seen as a model for generating unvalidated knowledge claims rather than knowledge (Firestone, 2000, 2001). Or that it fails to make the distinction between tacit, explicit, and implicit knowledge (Firestone, 2001, 2002, Ch. 7), and not just between tacit and explicit knowledge.

Regarding the term "knowledge," Snowden tells us that it was not problematic at the beginning of the first age, but became so at its end because the first age did not recognize the character of knowledge that was embedded in social interaction and in minds. In the first age, then, we can infer that Snowden thinks that the terms 'information' and 'knowledge' were used loosely relative to one another, if not interchangeably. Knowledge in the second age, however, took on some special meaning, although he never really tells us what that is. Its special meaning is somehow tied in with the SECI model, the touchstone of the second age. Still, none of this would seem to support the assignment of "age" status to either period in Snowden's account. Why? Because even if we can agree to include the pre-1995 period in which he describes the handling of information and not knowledge at all, these distinctions, from a generational point of view are, like Koenig's ideas, nothing more than "a story" about evolution in tools and methods. They do not point to evolution in the underlying conceptual or analytical frameworks of $\mathrm{KM}$ and knowledge processing, much less a distinction between the two.

The generational view, on the other hand, has a much easier time of accommodating the phenomena just mentioned without having to resort to the declaration of new ages, stages, or generations. Indeed, they are all mainly about information and/or knowledge transfer or integration. Add them to a long list of other techniques aimed at enhancing knowledge sharing or transfer, and you're still left with one stream of practice: supplyside KM. Thus the generational view comprises a much broader framework than the SECI model, in that it incorporates all of the varied activities of KM practice listed earlier in this paper as occurring since 1995. In fact, SGKM is broad enough to include much, perhaps most, of the aspects that are supposed to distinguish Snowden's third age from SGKM itself. We'll explore this and other problems in the next section on Snowden's forecast. 


\section{Snowden's Forecast: A Third Age of KM?}

Snowden (2002) contends that the third age will be one in which:

- Knowledge is viewed paradoxically as a thing and a flow,

- Context, narrative and content management will be central to our view of $\mathrm{KM}$,

- There will be an understanding of organizations as engaged in sense-making through utilization of complex adaptive systems phenomena constrained by human acts of free will attempting to order them, and finally,

- Scientific Management with its mechanistic models will be applied to carefully selected targets where it is appropriate, while the outlooks of Chaos and Complexity Theory will be applied to other targets and situations where they are appropriate.

There are a number of ways to look at this forecast. Let us start with its implicit factual claim that the present condition of KM is not characterized by the above attributes.

\section{KM and Scientific Management}

Is it true that KM does not now restrict Scientific Management and its mechanistic models to carefully selected situations where these may be relevant? We think the answer to this question is yes.

We don't know of a single writer on KM who endorses Scientific Management and its mechanistic models as the dominant approach to KM, and we know of many writers who explicitly reject the relevance of such an approach to most human-based interactions. These writers include: Allee (1997), Amidon (1997), Brown (1995), Brown and Duguid (2000, 1991), Carrillo (1998, 2001) Davenport and Prusak (1997), Denning, et. al. (1998), Denning (2001), Firestone (1999, 1999a, 2000, 2001), Kuscu, 2001, Leonard-Barton (1995), McElroy (1999, 1999a, 1999b, 2000, 2002 ), Senge (1990), Wheatley and Kellner-Rogers (1996), among many others. In short, it is simply false that third generation knowledge management, if there is to be one, will be unique in restricting mechanistic management models to only those situations in which they are relevant, since that restriction has already come to pass.

\section{KM, Content Management and Context}

Is it true that $\mathrm{KM}$ is not now characterized by context, narrative and content management, and that therefore this would be a distinctive development in a coming third age or generation of KM? In reviewing Koenig's views we 
have already pointed out that content management has been a concern in KM for some years now, and also that the 1998 combined KM World/AlIM conference held in Chicago had a major content management element. In fact, many vendors have long confused content management and knowledge management, as if there were nothing more to KM than that.

And since 1998, the ties between knowledge management and content management have grown stronger with the connection that is currently made between Enterprise Information Portals (with substantial content management capabilities) and knowledge processing and KM. In our view, that connection is greatly overdrawn (Firestone, 2002, Chs. 15 and 17). Still, from Snowden's point of view content management is at the heart of the third age of KM, even though it certainly has not been far from the attention of KM practitioners since the mid-90s. Snowden in fact recognizes the close connection between content management and $\mathrm{KM}$ prior to the third age. He says (2002, p. 101):

"Stacey accurately summarises many of the deficiencies of mainstream thinking, and is one of a growing group of authors who base their ideas in the science of complex adaptive systems. That new understanding does not require abandonment of much of which has been valuable, but it does involve a recognition that most knowledge management in the post 1995 period has been to all intents and purposes content management."

So clearly, content management is not a distinctive characteristic of any forecasted third age.

Regarding context, Snowden's (2002) use of that term is not transparent. Linked as it is with content management by Snowden, our first interpretation was that he was mainly referring to context analysis in the context of content analysis and management. But his primary concern with context instead comes from the notion that (ibid., p. 102):

“. . . human knowledge is deeply contextual, it is triggered by circumstance. In understanding what people know we have to recreate the context of their knowing if we are to ask a meaningful question or enable knowledge use. . . "

In other words, the knowledge people have that is directly related to knowledge use is the set of situational beliefs they use to perform acts. And these beliefs are not determined outside of situational contexts, but through the interaction of people with those contexts (or experiences). Moreover turning to Stacey (2001) - Snowden suggests that these beliefs (knowledge) are ephemeral, precisely because of their grounding in 
momentary experiences, or contexts. He seems to be saying that because experiences are fleeting, so, too, must knowledge evoked in the course of experience be fleeting. In any case, he is clearly suggesting that knowledge beliefs and use are at least partly determined as a function of situational contexts.

Now, as it happens, this last thought is not a new idea. It is one that has been well-known in the social sciences for many decades, and has certainly been well-known in social psychology for many, many years. Figure 2 illustrates the idea of an agent making decisions and engaging in transactions with other agents as part of a social network or system. The beliefs affecting behavior in the diagram are within the agent. The beliefs closest to behavior are the "sense" (Weick, 1995; Haeckel, 1998) that the agent has made out of the situation. The context is provided by the transactions directed at the agent and also by the social ecology box in the figure including its physical, social structural, and cultural components.

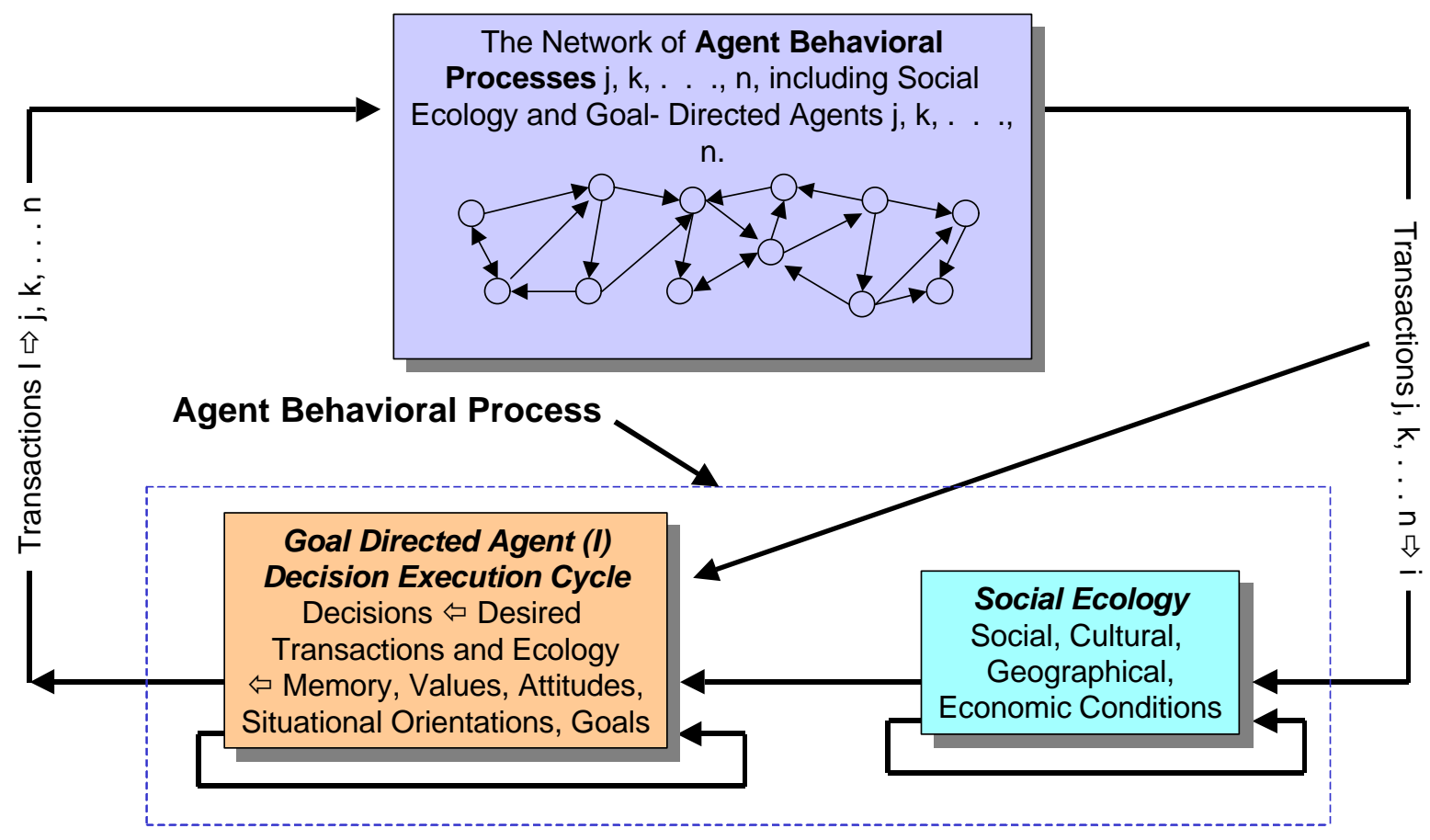

Figure 2 - The flow of behavior among agents

The process of interaction going on inside the agent may also be viewed as the Decision Execution Cycle of the agent, illustrated in Figure 3. In the figure, sense-making is represented by the step called "Monitoring." 


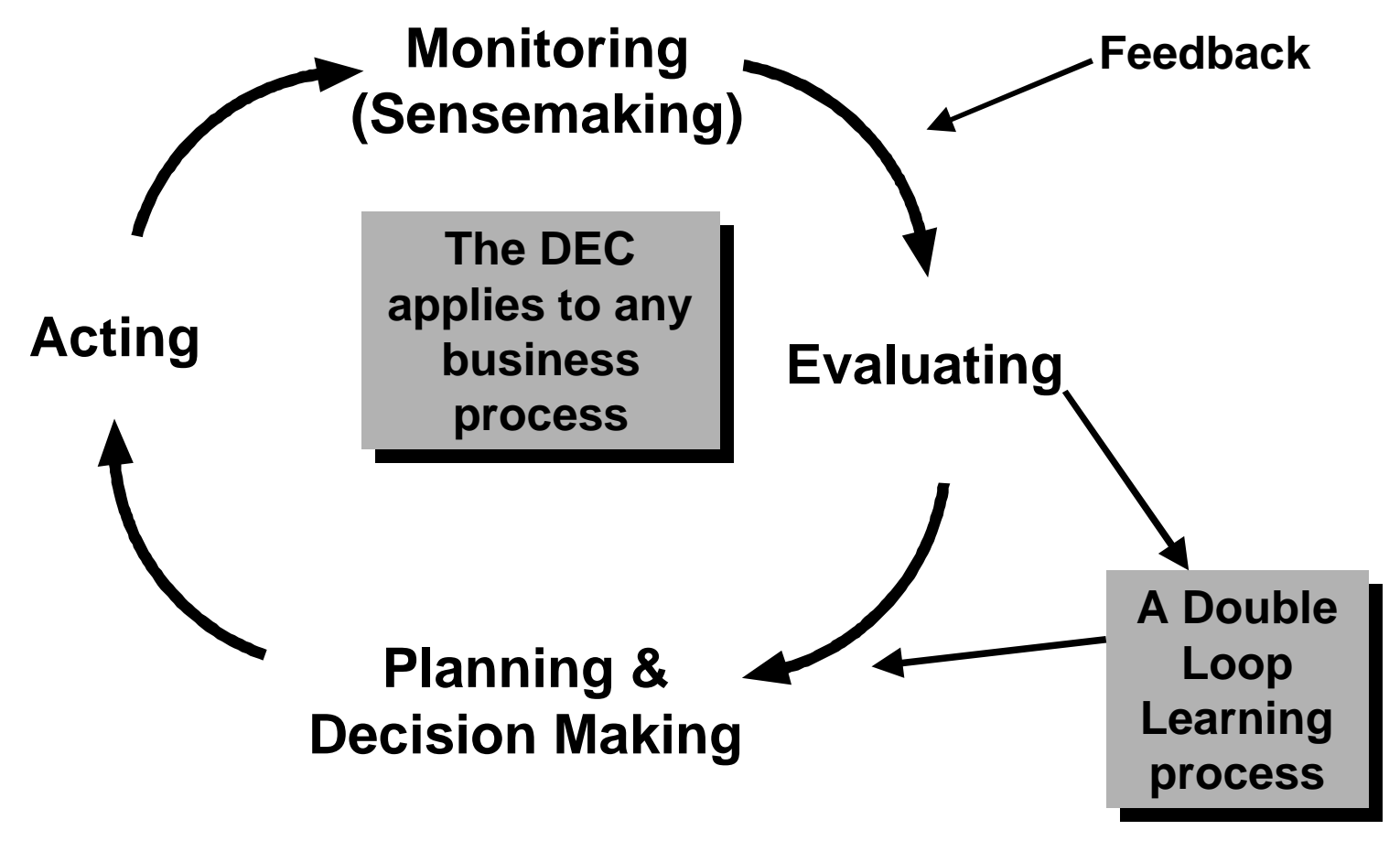

Figure 3 - The Decision Execution Cycle

Figure 4 provides the psychological context of the formation of situational knowledge and beliefs. It shows a hierarchy of psychological predispositions of any agent that are aroused by the external situational context, that form an internal psychological context, and that themselves affect the formation of contextual knowledge beliefs. This hierarchy, called an incentive system (Birch and Veroff, 1966), produces a situational orientation of the agent. The availability and incentives represent the "sense" the agent has made of the situation. The situational incentive refers to the affective orientation produced by the predispositional hierarchy toward the situation. The resulting behavior is the outcome of the goalstriving produced by the interaction of the availability, incentive, and affective components.

The notion presented in Figure 4 is oversimplified in that it ignores the overwhelming probability that behavior in any concrete situational context will simultaneously be motivated by more than one incentive system. A contemplated action, in other words, may be associated with a likely outcome having multiple and either conflicting or reinforcing incentive values or value expectations for an agent. So the depiction of a single goalstriving tendency at the bottom of Figure 4, just prior to the discrete situational orientation, is misleading. Instead, visualize a number of conflicting goal-striving tendencies, $G_{1} \ldots G_{n}$, all firing in parallel, and take 


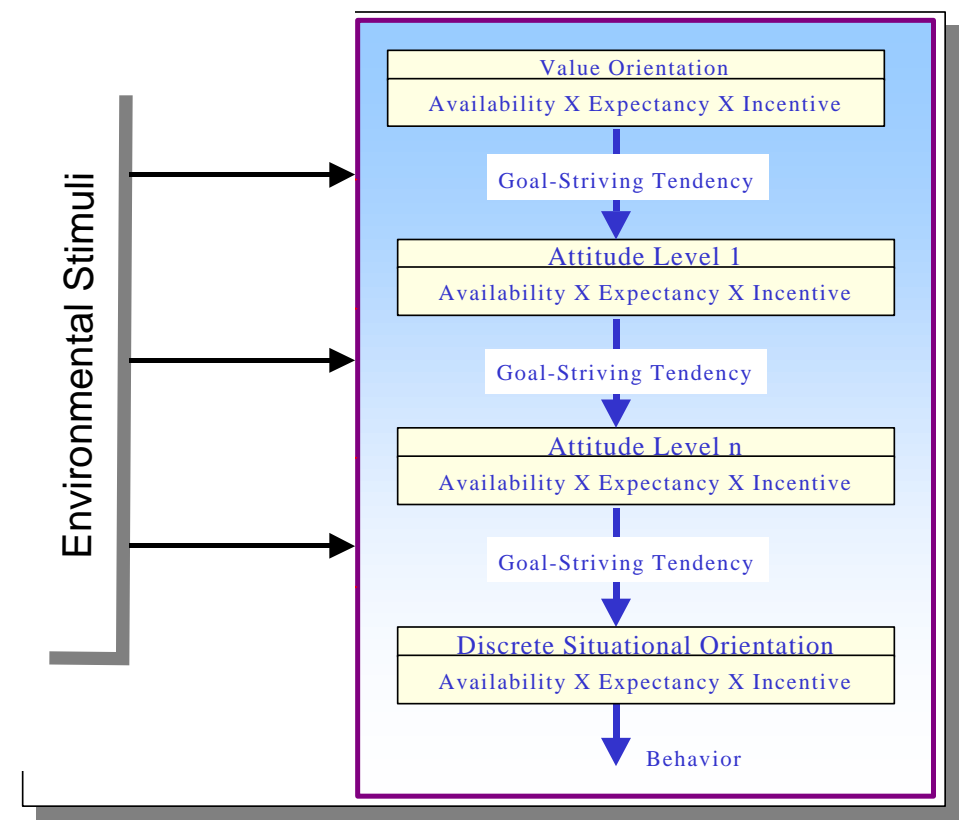

The psychological motivation of an individual or the culture of a group-level agent is defined by the value orientations, attitudes, and goalstriving tendencies in the box The environmental stimuli include social ecology and transactions

\section{Figure 4 - The incentive (motivational) system of an agent}

the resultant of these, along with the environmental stimuli, as affecting the discrete situational orientation.

This kind of conception of situational knowledge being formed in context is not new to Knowledge Management. One of us (Firestone [2001]) proposed this framework in an explication of the notion of "subjective culture" for use in second generation KM. His development of this framework is not connected to any fundamental generational change beyond SGKM, but only to an attempt to strengthen its foundation by clarifying the role of knowledge as belief or belief predisposition; knowledge as a cultural product; and culture, itself, in business, knowledge, and knowledge management processes.

If the notion of contextual knowledge is not, as Snowden implies, new to $\mathrm{KM}$, is it true that his conceptual development of the idea of context, combining abstraction and culture is significant enough that it should form the basis of a reorientation of $\mathrm{KM}$ and therefore a third age? We believe that the answer to this question is clearly 'no' because:

- descriptions of context in terms of abstraction and culture are much too simple without much more detailed development of the framework, 
- context alone neither comprises nor determines knowledge production and integration processes, and

- a revision in the idea of how to categorize context for purposes of description and analysis is not the kind of fundamental change in orientation that signals a new generation, or a new age.

Thus, why wouldn't such a change just fit into the second age? It may not do so, if one defines the second age, as Snowden does, as essentially one in which activity is focused on the SECI model, but if one takes the broader SGKM or TNKM point of view, changes in how we categorize or describe context for the purpose of affecting knowledge production and knowledge integration are just "par for the course" and involve nothing more than further development of the TNKM point of view, rather than a departure from it into a new generational outlook.

\section{Knowledge: Process or Outcome?}

Is it true that knowledge is not now viewed paradoxically as a thing and a flow and that this view needs to be adopted in order to get past the difficulties associated with viewing knowledge as a thing? Our answer to this challenge is to agree that "knowledge" is not now viewed paradoxically as both a thing and a flow, but also to state unequivocally that to adopt such a view would not solve the problems of knowledge management, but rather would only deepen the degree of confusion and conflicts existing in the discipline.

Let us now examine our reasons for this conclusion by offering responses to some of Snowden's comments on the nature of knowledge as a process and then both a "thing" and a "flow." Snowden says (2002, p. 101):

"Some of the basic concepts underpinning knowledge management are now being challenged: "Knowledge is not a "thing", or a system, but an ephemeral, active process of relating."

Taken from Stacey (2001), this definition suffers from, or at least creates, a process-product confusion. It is fueled by a desire to focus on the dynamics of knowledge creation, rather than only on explicit codified outcomes or mental beliefs. However, we can do this without becoming confused just by distinguishing knowledge processes from knowledge products or outcomes. Knowledge processes are not any less important because we call them "knowledge processes" rather than "knowledge" (the "ephemeral active process of relating"). 
Why should we avoid the process-product confusion? First, if we take the view that knowledge is a process, we can no longer talk about knowledge as embedded in cultural products, or even knowledge as beliefs or predispositions in minds. Or knowledge as "true" or "false," since processes are neither true or false, but only existent or non-existent.

Next, if we tolerate the confusion, it doesn't allow us to account for the content of cultural products or beliefs or predispositions in minds. So we are left with the problem of finding words other than knowledge to describe these very real phenomena. The real question is: what do we gain by calling knowledge "an ephemeral, active process of relating"? What does it do for us? In our view it only adds confusion in a field that is already replete with it, because some people insist on using words for their "halo effect" rather than for their descriptive value.

To us, it seems clear that knowledge is not a process but an outcome of knowledge production and integration processes. In other words, we believe that knowledge should be viewed as a "thing," not a process. We also believe that as specified elsewhere (Firestone, 2001), knowledge is not a single thing, but is divided into three types: physical, mental, and cultural. All are things, and more specifically are encoded structures in systems that help those systems respond and adapt to changes in their environments.

Next, Snowden says:

“. . mainstream theory and practice have adopted a Kantian epistemology in which knowledge is perceived as a thing, something absolute, awaiting discovery through scientific investigation." (ibid.)

To say knowledge is a thing may be Kantian, or sometimes even Platonist for that matter, but to label it in this way is not to criticize the idea on its merits. Furthermore, to say that knowledge is a thing is not to say that it is "absolute," or that it is "awaiting discovery through scientific investigation." That is, knowledge can be (a) a thing, (b) produced by social processes of many kinds, and not just processes of scientific investigation, much less awaiting discovery by the latter, and (c) can also be either false or true. So there is nothing "absolute" about it.

Snowden also says:

"In the third generation we grow beyond managing knowledge as a thing to also managing knowledge as a flow. To do this we will need to focus more on context and narrative, than on content." (ibid.) 
As far as the third generation (or age) being about managing knowledge as a flow is concerned, if by "flow" Snowden means knowledge processing, then we do not agree that this is distinctively third generation, but instead think it is second generation $\mathrm{KM}$ and is at least a few years old now, as our discussion of SGKM above indicates. But is this, in fact, what he means by 'flow'?

Again, Snowden says:

"Properly understood knowledge is paradoxically both a thing and a flow; in the second age we looked for things and in consequence found things, in the third age we look for both in different ways and embrace the consequent paradox." (ibid., p. 102)

Here we see a shift in Snowden's view. As we saw above he begins by characterizing knowledge as a process and creating a process-product confusion, but ends by claiming that it is both a "thing" and a "flow," thereby creating a process-product redundancy (to wit, flows are things). This he denies is a redundancy, treats as a seeming contradiction, and terms a "paradox." He then defends paradox, by pointing out that philosophers have learned much from paradox, and also that physicists have had to live for many decades with the paradox that electrons are both particles and waves.

This is all very neat, but it is also very problematic: (1) Philosophers have learned much from paradox, but this doesn't mean that paradox in the definition of knowledge is necessarily good for $\mathrm{KM}$, especially if there is no paradox. (2) It is not true that physicists have concluded that electrons are both particles and waves. Rather, electrons are things that may be described using a particle model under certain conditions and a wave model under others. The reason why there is no contradiction or paradox in this view is that physicists know enough not to claim that electrons are both waves and particles, but that they are a third thing entirely. Indeed, this is the key lesson embodied in the Heisenberg Principle.

And (3), and most importantly, Snowden hasn't established the need to call knowledge both a thing and a flow and thereby embrace paradox, contradiction or redundancy, much less another age of KM founded on paradox. All we need do, instead, as we in fact have done in SGKM, is to say that knowledge is an outcome or product (thing) that is produced by human social processes (process). Thus, we have the ability to deal with both dynamics and outcomes in such a conceptualization, an ability that has always existed in systems theory. 
So, the effort to establish knowledge first as a process, and then as a "thing" and a "flow," is not persuasive to us. It seems to offer no advantages that the process-product view of SGKM is not already delivering. On the other hand it offers the disadvantages of logical contradiction, redundancy, or perhaps paradox, if one accepts Snowden's assertion, that can only lead a third generation founded on it into unnecessary confusion and perplexity. Our conclusion is that we don't need such a third generation, but that what we do need is to continuously tighten the conceptual foundations of SGKM and continue to develop its program of research and practice.

\section{Sense-Making, Complex Adaptive Systems, and the Third Age}

Is there already an understanding in Knowledge Management of organizations as engaged in sense-making through utilization of complex adaptive systems phenomena constrained by human acts of free will attempting to order them? Or is this a distinctive feature that might provide the foundation for a third generation of $\mathrm{KM}$ ?

Recognizing the role of complex adaptive systems phenomena in human organizations, "sense-making," and knowledge production is very important in understanding the emergence of organizational behavior, organizational knowledge predispositions, organizational learning, and organizational intelligence from interactions among organizational agents. But whether or not recognition of the importance of CAS phenomena, and their interaction with purposeful knowledge management interventions creates the need for a new KM generation depends very much on one's view about previous generations.

If you accept Snowden's view that the second age of $\mathrm{KM}$ is about knowledge conversion and the Nonaka/Takeuchi program alone, and that it (a) did not focus on knowledge processing, (b) had no emphasis on the situational character of knowledge, (c) was committed to the development of mechanistic models of knowledge management, and (d) did not recognize the role of CAS in knowledge processing and knowledge management, then to declare the need for a new generation may make sense. But if you view SGKM, as we do, as a professional discipline developing since 1995 to:

1. emphasize the distinctions among knowledge, sense-making, knowledge processing, knowledge management, business outcomes, business processing, and business management,

2. add a focus on knowledge production (rather than just knowledge conversion) and sustainable innovation to a previous focus on knowledge integration, 
3. arrive at a conceptual framework that emphasizes the situational character of sense-making and belief knowledge; that breaks knowledge production and knowledge integration into subprocesses; that identifies knowledge management activities and their targets in knowledge processing; that makes clear the link between knowledge processing, explicit knowledge production, belief knowledge production, and knowledge use; and that relates all of this to the situational context of sense and decision making and organizational learning cycles,

4. recognize patterns of knowledge processing that emerge from CASbased interaction tempered by KM initiatives,

5. recognize that $\mathrm{KM}$ initiatives must be synchronized with CAS phenomena in order to succeed,

6. deny the relevance of mechanistic management models for knowledge management,

7. emphasize the central role of knowledge claim evaluation (or validation) in $\mathrm{KM}$,

8. emphasize the important role of communities of practice in mobilizing CAS-based interaction and contributing to both knowledge production and knowledge integration,

9. recognize the role of culture in providing a context for knowledge processing and knowledge management,

10. emphasize a coherent theory of knowledge that distinguishes it from data, information, and wisdom,

11. develop a systematic approach to knowledge and KM-related metrics,

12. place the role of information technology in context as an enabler of knowledge processing and $\mathrm{KM}$ processing,

13. recognize a model of Intellectual Capital that sees social innovation processes as an aspect of such capital, and

14. develop and use methodology that incorporates all of the above elements and that is oriented toward problem-solving,

then you may feel that everything that is distinctive and useful in Snowden's forecasted third age already exists in the second generation, that is, in TNKM. So from the point of view of TNKM, there is no third age nor any need for one.

But even if all of the above is correct, what about Snowden's Cynefin model, doesn't it suggest that a third age is upon us?

\section{The Cynefin Model and Its Problems}

Our treatment of the Cynefin model will be detailed and follows the following pattern: We summarize a bit of the model and then present commentary and criticism. We then repeat this pattern until the analysis is 
complete. We then offer a summary of the whole discussion and some general perspectives.

The Model: The Cynefin model uses the distinctions between the poles of the context dimensions (high and low abstraction, and teaching and learning cultures) to initially create four types. In Snowden's words (2002, p. 104)

"Cynefin creates four open spaces or domains of knowledge all of which have validity within different contexts. They are domains not quadrants as they create boundaries within a centre of focus, but they do not pretend to fully encompass all possibilities."

The Cynefin Model not only specifies four open spaces or domains of knowledge. It also views those spaces (See Figure 5) as "Common Sense Making" environments.

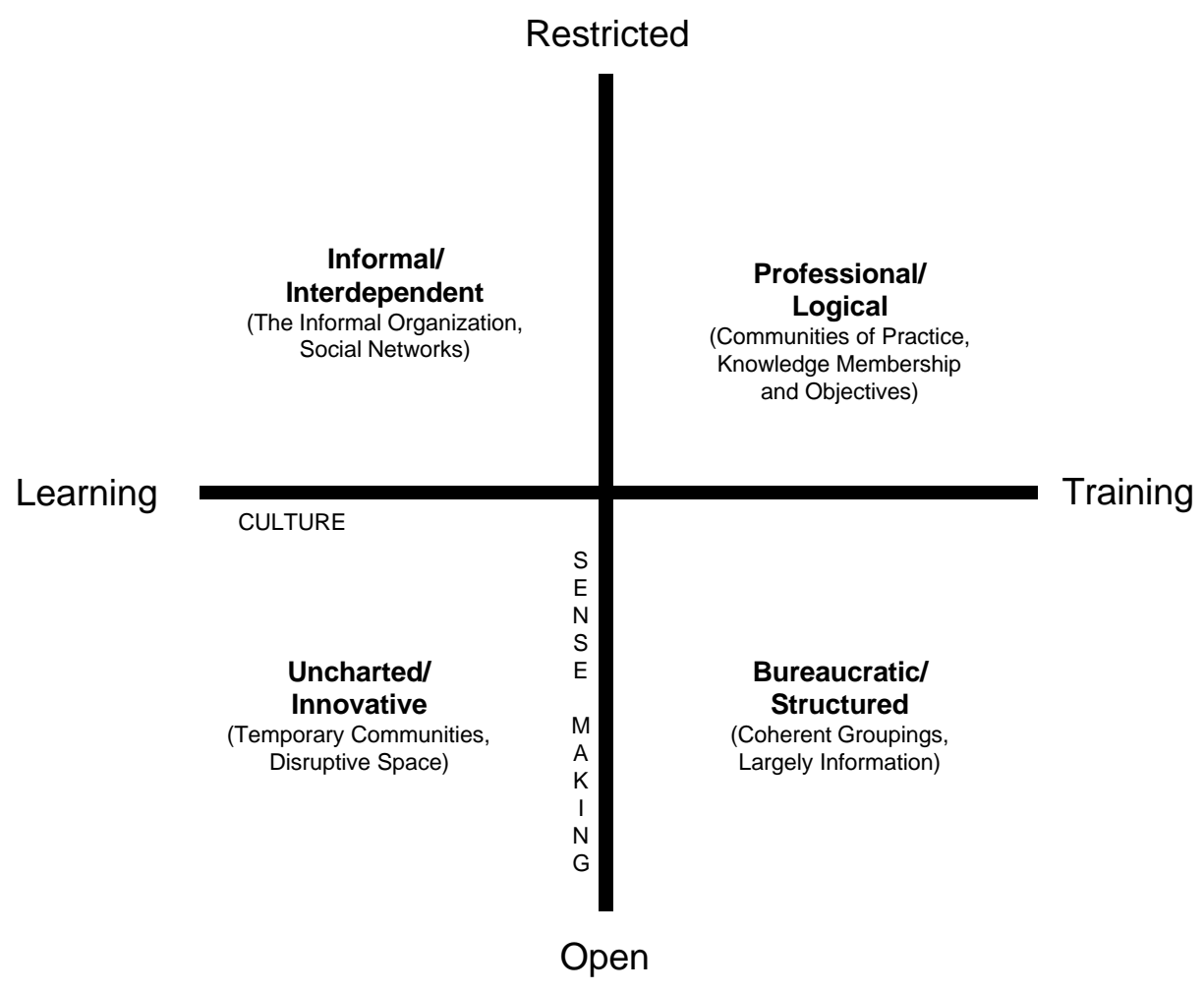

\section{Figure 5 - Cynefin Model: Cultural Sense Making}

(Source: A Replica of David Snowden's Cynefin Model) 
Commentary: The first problem with the Cynefin Model is with the specification of the two context dimensions used to formulate it: culture and abstraction.

Snowden bases his concept of culture on Keesing and Strathern's (1998) work. They distinguish between the socio-cultural system (what people do and make) and the ideational system (or what people learn) as two different types of culture. Snowden, however, also notes that the first type of culture is teaching culture and the second type of culture is learning culture. In forming the Cynefin model, he then uses the dimension teaching/learning to define an aspect of variation among sense-making environments.

Now, the problem with this is the gross oversimplification of the Keesing/Strathern distinction between the two types of culture. The two types are based on numerous dimensions in Keesing and Strathern, not on the distinction between teaching and learning. To suppose that the teaching/learning distinction is all that is meaningful in the notion of culture for specifying sense-making environments is to make a wildly optimistic and obviously incorrect assumption.

The second context dimension (high/low abstraction) is also specified inadequately by Snowden. Snowden (2002) does not define what he means by abstraction. In Snowden (2000, p. 245) he comes closer to defining abstraction in the following passage:

"Such communitites are working at a high level of abstraction. Abstraction is the process by which we focus on the underlying constructs of data. As Boisot (1998) admirably demonstrates, the process of abstraction is focused on concepts, not percepts. Percepts, '....achieve their economies by maintaining a certain clarity and distinction between categories, concepts do so by revealing which categories are likely to be relevant to the dataprocessing task" or information creation. 'Abstraction, in effect, is a form of reductionism; it works by letting the few stand for the many.'"

But this definition of "abstraction" is still unclear. Specifically, we now know that abstraction is a process, but we don't know (from Snowden's account) what we actually do when we abstract and we don't know what "high abstraction" and "low abstraction" mean to Snowden in sense-making environments. We do receive the further information in both Snowden articles that level of abstraction is inversely related to cost of disembodiment or codification. But this idea seems to imply that abstraction is an outcome (actually an attribute of information) rather than a process, as Snowden has designated it in the above quote. In short, we don't know 
what Snowden means by abstraction. So it's very difficult to evaluate his description of common sense-making environments in terms of high/low abstraction.

The above considerations immediately call into question the Cynefin model. If we have no clear idea of what is meant by "abstraction" and if the distinction between teaching and learning cultures oversimplifies contextual variations due to culture, then how are we to understand the relation of these dimensions to common sense-making environments?

The Model: In Snowden's Figure 2, the domains are labeled "Common Sense-making," so that each of the four constitutes a distinctly different environment for sense-making.

Commentary: Snowden's immediate purpose in constructing Cynefin was to specify four distinct sense-making environments that sensemakers encounter in their everyday experience in organizations. But is sensemaking really the primary goal of knowledge processing? And is it the same thing as knowledge production?

Undoubtedly, sense-making is an important activity. According to Figures 2, 3, and 4, sense-making is a critical step in the Decision Execution Cycle (DEC) underlying all action and all business process behavior including knowledge process behavior. But sense-making, business processing, and knowledge processing are not equivalent. This lack of equivalence raises the question of where sense-making stands in relation to knowledge management. Knowledge management is management of knowledge processing and its immediate knowledge outcomes, and business management is management of business processes, generally, and their outcomes. Since sense-making is a part of all business process behavior, it falls under the general purview of business management rather than knowledge management, unless the sense-making in question is specifically tied to Decision Execution Cycles comprising the various subprocesses of knowledge production and knowledge integration.

This argument raises the question of the specific relevance of a sensemaking model such as Cynefin to knowledge processing and knowledge management. Still, it has some relevance to knowledge processing and knowledge management. Both areas are areas of business process behavior and so both, at the lowest level of decision making and acting involve sense-making. But sense-making is only one activity in the DEC, and, in addition, patterns of sense-making don't speak directly to the dynamics of knowledge sub-processes such as information acquisition, individual and group learning, knowledge claim formulation, and knowledge claim evaluation, and the various knowledge integration sub-processes. So at most even a good model of sense-making would not be broad enough in 
its relevance to define the scope of knowledge management in its third age.

On the other hand, since sense-making relates to all decision making through the DEC, a good model of sense-making will add to our theoretical and practical understanding of the actions that are the foundation of knowledge processing and knowledge management. So sense-making models certainly have a place in providing a better understanding of the decision making foundation of SGKM processes.

The Model: Here is the first of the four environments:

- Bureaucratic/Structured; teaching, low abstraction

This common sense-making environment emphasizes formal organization, policies, rules, procedures and controls. Snowden emphasizes the explicit, open nature of language, training, and the corporate intranet as important features. He also points (2002, p. 104) out that "its shared context is the lowest common denominator of its target audience's shared context." In other words the level of abstraction characterizing the shared context of communications is low.

Commentary: Here, Snowden does not explain why an ideal type of Bureaucratic/Structured, learning and high abstraction would not be equally useful as a common sense-making environment. Or for that matter why the other two ideal type variations based on the teaching/learning and high abstraction/low abstraction dichotomies, Bureaucratic/Structured, learning and low abstraction, and Bureaucratic/Structured, teaching and high abstraction, should not also be selected. In other words, he provides no explanation why he settled on the above pattern as the only sense-making environment within the Bureaucratic/Structured category. In other words, he doesn't explain the logic behind his specification of the above specific Bureaucratic/Structured sense-making environment as one of his four primary types of common sense-making environments.

Here is the second of the four environments:

\section{The Model:}

- Professional/Logical; teaching, high abstraction

This common sense-making environment is characterized by a high level of abstraction in the shared context for communications. It is also characterized by professional individuals, expertise, training, specialized terminology, textbooks, communities of practice, and "efficient knowledge communication" especially among experts. 
Commentary: A similar comment can be provided here as we offered for the Bureaucratic/structured types. Why specify teaching/high abstraction along with Professional/Logical? Could not Professional/Logical sensemaking environments involve both learning and low abstraction as well as teaching and high abstraction? Or other combinations of these categories? Of course they can. Do other combinations make sense? We don't know, but we do know that the rationale presented by Snowden for the type he prefers is not clear to us and, we suspect, to other readers as well.

Here is the third of the four environments:

\section{The Model:}

- Informal/Interdependent; learning, high abstraction

This environment has a high level of abstraction in the shared context of experiences, values, symbol structures, and beliefs. It is focused on the informal organization and its "network of obligations, experiences and mutual commitments." It is also characterized by trust, voluntary collaboration, story-telling, the ability of symbolic languages to efficiently convey large amounts of information through reliance on highly abstract symbol associations and shared symbol structures. This information can include (ibid.) "simple rules and values that underlie the reality of that organization's culture (Snowden, 1999)."

Commentary: Again, the pattern specified by Snowden is not the only pattern that can be specified for Informal/Interdependent sense-making environments. In particular, we think that low abstraction in the shared context of experiences, values, symbol structures etc, is also possible in such environments, as is teaching.

Further, the specification that an Informal/linterdependent environment is characterized by trust and voluntary collaborations is certainly only one possibility. Informal/Interdependent sense-making environments may also be characterized by mistrust and socially coerced collaboration, as well. Of course, such a sense-making environment may be less effective at sensemaking than the one specified by Snowden. But we don't know that yet based on research, while we do know that expectations based on simple ideal types are often frustrated by complex reality.

Here is the fourth of the four environments:

\section{The Model:}

- Uncharted/Innovative; learning, low abstraction 
This environment presents entirely new situations to an organization. It is "the ultimate learning environment (Snowden, 2002, p. 105)," and is characterized by low abstraction in the shared context of communications among agents in this common sense-making environment. Snowden says (ibid.):

"Here we act to create context to enable action, through individuals or communities who have either developed specific understanding, or who are comfortable in conditions of extreme uncertainty. Such individuals or communities impose patterns on chaos to make it both comprehensible and manageable."

Commentary: Is this really the perfect learning environment? Why would one think that one can learn better without context than with it? This would be true only if one assumes that context is always more constraining than chaos. But certainly this is not always true. In a very real sense, chaos may be the best unlearning environment, the opposite of what Snowden suggests. Further, why is the environment characterized by low abstraction? If it is truly uncharted, then decision makers can create their own context, with a level of abstraction appropriate to them.

The Model: Snowden tells us that the Cynefin model we have just outlined is based on the distinctions among chaotic, complex, and complicated systems. By complicated systems he means those systems whose cause and effect structure is either known or knowable. By complex systems he means those with coherent structures and processes whose cause and effect structure cannot be known and whose global behavior is emergent, but which is not explainable in terms of a system's components and their relationships. By chaotic systems he means those systems in which "all connections have broken down and we are in a state of turbulence or eternal, boiling." (2002, p. 106)

Commentary: We believe that this typology of systems is incomplete, and that human social systems are not Natural Complex Adaptive Systems (NCASs), such as insect social systems, but Promethean Complex Adaptive Systems (PCASs). That is, we find CAS behavior in them, but such behavior is moderated by the continuous efforts of human agents to create predictable structures that serve their interests. These efforts use normative processes that attempt to simulate cause and effect sequences by treating humans as if they are objects that will respond to prescribed stimuli in prescribed ways. However, the behavioral processes corresponding to these normative processes are not processes in complicated systems, rather they are "complex" processes, always subject to human adaptation and innovation in the face of changing conditions. So PCASs follow neither CAS, nor complicated, nor chaotic patterns, but 
rather their own patterns that oscillate constantly between different states of relative complexity.

Also, we need to note at this point that the boundary between complicated and complex systems is not hard and fast in the sense that it is obvious when a system belongs to one class or the other. To be a CAS or a PCAS, rather than a complicated system, it is necessary that a system not be "knowable." However, a system that today seems unknown or unknowable, may tomorrow be knowable or known. So we can never say for certain that a particular type of system fits into one category or another.

The Model: After introducing these distinctions, Snowden asserts that these three system types "map on to the Cynefin model." (ibid.) Chaotic systems map on to the Uncharted/Innovative Common Sense-making Environment, Complex systems map on to the Informal/Interdependent environment, and Complicated systems map on to either the Professional/Logical or Bureaucratic/Structured environments, depending on whether the targets of decision making are "knowable" complicated systems, or "known" complicated systems.

Commentary: Snowden does not explain the above mappings and it's not at all obvious that they "make sense," or even what he means by "mapping." Does he mean to say that the Bureaucratic/Structured sensemaking environment is a known system? Or that the Professional/Logical sense-making environment is a "knowable" system? Or that the Informal/Interdependent sense-making environment is a complex system? Or that the Uncharted/Innovative sense-making environment is a Chaotic System? If that is the meaning of "mapping," it seems invalid on its face, because all organizational sense-making environments are part of a single system, the organizational system. And that system is a type that we have previously called a PCAS. Its nature cannot be changed by interpreting sense-making environments as though they were autonomous systems without risking serious misunderstanding of the dynamics of the organizational system.

Indeed, Stacey (1996, p. 184), himself, makes this point in the following passage in which he discusses the possibility (or not) of human agents being able to separate themselves from the organizational environments of which they are a part in their attempts to manage the creative dynamics of human social systems:

"Do human consciousness and self-awareness somehow enable us to alter the dynamics of the systems that we are a part of and that we constitute with each other when we interact? 
At the level of the organization, as at the levels of the individual mind and the group, the answers to these questions must be no. First, it is extremely difficult for members of an organization to sustain enough emotional distance from their roles as participants to also operate as observers.... . . Even when we manage to reflect collectively on the organization we are a part of, we are still not able to alter the fundamental dynamics of that system."

An alternative interpretation, and the one we favor because it is most in accord with the interaction philosophy at the base of sense-making (see Weick, 1995, Smythe, 1997), is that Snowden is seeking to correlate his previous construction of sense-making types in the Cynefin model with the types of target systems that each of these environments is most suited for, in terms of the likely success of sense-making activities in these environments in "making sense" of the target systems and successfully dealing with them over time. In other words, this second interpretation is that the sense-making environment Snowden is talking about is comprised of the interaction between a Cynefin sense-making type and one of the four types of target systems he specifies. Such a relationship is illustrated in Figure 6. But even though this interpretation initially seems most plausible, further consideration indicates that it too would indicate an error by Snowden.

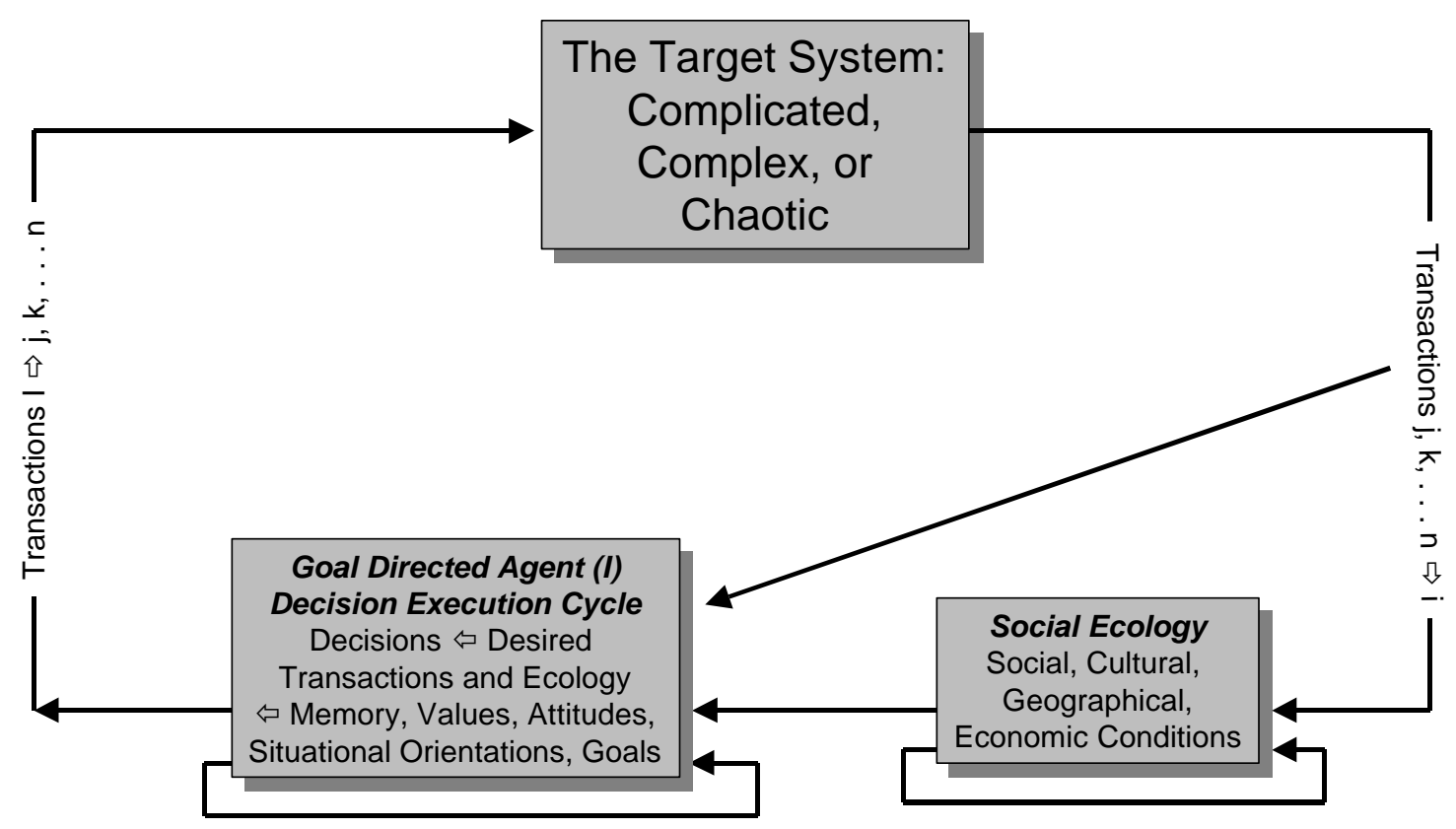

Figure 6 - An interactive sensemaking environment 
Why should we, for example, use an uncharted, innovative sense-making environment to make sense of a chaotic system? It seems to us that any sense-making environment would work, so long as through use of it we can recognize that the system that is the target of our sense-making efforts is, in fact, a chaotic system. Similarly, why should an informal, interdependent sense-making environment be necessary to decide that a target system we are interested in is, in fact, a complex system?

So if the second interpretation is correct, Snowden seems to have fallen prey to a subject/object confusion of the organization and the target system that is the object of its sense-making. If that is true, then no mapping of the type of the target system to the type of sense-making environment can be taken at face value, and Snowden needs to explain why other mappings of sense-making environments to target systems (e.g. Professional/Logical to Complex Systems) are not equally valid.

The Model: Snowden next develops the Cynefin model by associating various characteristics with the four Cynefin sense-making environment/ system type combinations. Known space (the domain of bureaucratic structured/teaching/low abstraction/known systems) is associated with best practices, the ability to predict behavior, to prescribe specific policies, and a "feudal" leadership style. Snowden also thinks that people can transform complex or chaotic systems into known systems "through laws and practices that have sufficient universal acceptance to create predictable environments." (Snowden, 2002, p. 106) Once these known systems are created, decision making can proceed by categorizing incoming stimuli and responding "in accordance with predefined procedures." (ibid.)

Commentary: Why should "known space" be associated with a feudal leadership style? Evidently this hypothesis is based on the notion that since the target system in known space is complicated, one needs a Bureaucratic/Structured sense-making environment governed by feudal leadership because this is the only type of sense-making environment capable of understanding a complicated known system. Surely this is an instance of the confusion of subject and object in mapping the original Cynefin model onto the systems typology used by Snowden.

Snowden also thinks that known systems can be created from chaotic or complex ones through instituting laws, policies, procedures, etc. All we have to say to that hypothesis is "good luck." Known systems cannot be created by fiat. Rather, a known system is a real system that is successfully described by some cause and effect theory we have developed. If no such cause and effect structure can be formulated, then normative human social processes cannot substitute for such a structure. Rather, the result of imposing laws and structures on complex systems is a 
Promethean CAS, not a known system. More likely, it's a dysfunctional PCAS.

As for the idea that chaotic systems may be transformed through leadership into knowable or known systems, this idea is also confused. That is, if a system is really a chaotic system, there are no cause and effect relationships that are understandable within it. So how can human agency have any predictable effect on such a system?

The Model: Knowable space (the domain of professional/logical/ teaching/high abstraction/knowable systems) is associated with good practice, expert explanations and predictions of behavior, expertiseenabled management by delegation, codification of expert language, entrainment of thinking, oligarchic leadership based on community "elders," and sensing and responding based on expert understanding. In knowable space, as in known space, humans impose order. But here the order is more "fluid" than in known space. To manage this space, it is necessary to periodically disrupt the body of expert knowledge and shared context, because they can be a barrier to the creation and growth of new knowledge.

Commentary: Why is "knowable space" associated with "entrainment of thinking" to a greater extent than known space? Are Professional/Logical environments more vulnerable to closed-mindedness than Bureaucratic/ Structural environments? We doubt it. Why is oligarchic leadership necessary to make sense of knowable space? Clearly, it's not. Why should "knowable space" imply that Professional/Logical sense-making environments using experts rather than Informal/Interdependent sensemaking environments using knowledge workers are necessary to make sense of them?

Why are periodic disruptions of the social structures of knowable space necessary to ensure continued effective sense-making in this space? The need for periodic disruptions is connected to the assumption that the content of knowledge is determined by an elite that supports a dominant paradigm that in turn controls the growth of knowledge. But what if knowledge production in an organization doesn't work that way? What if knowledge claim evaluation works through continuous testing and evaluation and openness in the evaluation process? What if knowledge is not developed based on consensus, but emerges through a continuous and open evaluation and testing process. This Open Enterprise model of knowledge production (McElroy, 2003, Chapter 1; Firestone and McElroy, 2002) would not need periodic disruptions to function well, because it is always open to new ideas. In fact, disruption would not improve knowledge production in such a system. Instead, it would disrupt the functioning of the open knowledge production process and might result in a system of 
Kuhnian paradigms (Kuhn, 1970) supported only by consensus, a system that would require periodic disruption.

The Model: Complex space (the domain of informal/interdependent/ learning/high abstraction/complex systems) is associated with selforganization, global pattern emergence, fluidity, stability at the edge of chaos, and emergent leadership based on natural matriarchal or patriarchal authority and respect. Here, according to Snowden, managers should recognize pattern formation early and manage patterns by stabilizing some and disrupting others based on goals, objectives, and values. They may even "seed the space" in hopes of encouraging desirable patterns. But prediction of emergent patterns is not possible in complex space. In this space agents cannot sense and respond. They must probe first to stimulate and/or understand patterns. Only then can they sense and respond successfully.

Commentary: We agree that leadership in complex space is emergent, but it is also true that in a PCAS emergent leadership must contend with imposed leadership. Also, what is the significance of the comment that emergent leadership in complex space is matriarchal or patriarchal? Is Snowden saying something more here than that such leaders may be either female or male? If so, what?

Also, note the very Promethean tenor of Snowden's comments about complex space. The idea that we can disrupt some patterns and reinforce or stabilize others, and even seed still others suggests scientific management. Moreover, since according to Snowden, we cannot predict emergent patterns in complex space, our ability to predict the outcome of our Promethean interventions is also problematic.

Even if we probe first "to stimulate and understand patterns" and then sense and respond, we don't know whether or not our response will stimulate emergent responses from the system that are unintended. In other words, the possibility of emergent side effects in complex systems suggests care in intervening and a search for as much cause and effect, statistical, and expert assessment knowledge about the system as we can muster. We should always keep in mind that something we've categorized as a complex system, may not, in fact, be one. Continued attempts to analyze complex systems as if they are "knowable" are therefore rational, if only to establish the degree to which they are not knowable.

The Model: Chaos (the domain of uncharted/innovative/learning/low abstraction/chaotic systems) is associated with lack of structured linkages among components, unpredictable, unfathomable connectivity among these components, tyrannical or charismatic leadership. According to Snowden, chaotic systems require active crisis management, lead to the 
disruption of entrained thinking in managers, require regular immersion to "immunize" organizations against chaotic systems, and can be used to advantage if leadership can impose order without loss of control. Snowden also thinks "that what to one organization is chaotic, to another is complex or knowable." Management must proceed in this domain by acting, and only then sensing and responding.

Commentary: Why do chaotic systems require active crisis management? Such systems cannot be understood and their behavior cannot be predicted. That is their nature. So why should crisis management of such systems work? Also, how can we immunize ourselves against chaos by immersing ourselves in such systems? Each chaotic system is unique and lacks a cause and effect structure. Would repeated exposures to multiple chaotic systems make the next chaotic system any less chaotic or unpredictable? We don't think so. So how can familiarity with them help us to cope?

Snowden also seems to believe in the relativity of chaos to the perspective of the organization beholding it. But this is certainly an unfortunate way of speaking. Surely, systems are either chaotic or not. It is our models of real systems which may vary, so that sometimes we mistake complex or knowable systems for chaotic ones. And, therefore what we thought was chaos is either at its edge or even orderly. Finally, does it really help management, as Snowden suggests, to act before sensing and responding to chaotic systems? If they really lack causal structure and are not subject to emergent patterns, then how can our acting first result in a better foundation for sense-making and responding? Such systems should be equally unfathomable and uncontrollable regardless of how we proceed through time.

The Model: A central tenet of Snowden's third age proposal is his contention that knowledge will be viewed, paradoxically, as both a thing and a flow. In his first reference to knowledge as flow, Snowden states that "Complex adaptive systems theory is used to create a sense-making model that utilises self-organising capabilities of the informal communities and identifies a natural flow model of knowledge creation, disruption and utilisation." (Snowden, 2002, p. 100). Later on in his discussion of the first age of KM prior to 1995, he says that the focus was on "the appropriate structuring and flow of information to decision makers." (ibid.) Next, in what appears to be a reference to flow he quotes Stacey (2001) as saying "Knowledge is not a 'thing,' but an ephemeral, active process of relating." (ibid. p. 101).

Later on, Snowden refers to flow in terms of the movement of knowledge in his discussion of the dimension of Abstraction in his Cynefin model as follows: "The upper and lower levels represent the range of shared context 
and therefore the range of possible knowledge flow." (ibid. p. 103). He goes on to say that both forms of culture he depicts in his model are "key to the flow of knowledge within an organization. We need to transfer to new members, in both the society and the organization, knowledge that has been painfully created at cost over previous generations." (ibid.)

Finally, in a subsection of his paper entitled "The Natural Flow of Knowledge," Snowden says "We can now see the sensible pattern of flow of knowledge within an organization," a claim he makes following his presentation of a view of the Cynefin model in which flows are depicted between his four domains. He summarizes his perspective on knowledge flow as follows: "From this perspective we see knowledge as flowing between different states, with different rules, expectations and methods of management." (ibid. p. 110)

Commentary: Given the importance of the view of knowledge as 'flow' to both Snowden's third age and the Cynefin model, it is critical to understand what he means by the term, and why he claims it is paradoxical in relation to the view of knowledge as a 'thing.' Earlier we noted the confusion caused by this language by pointing out that flows are things. Putting that aside, however, we fail to see either the claimed contradiction between the terms in this case, or the paradox between them.

To say that knowledge is something which flows, as most of his statements above would suggest, is not to invoke a contradiction at all or even a paradox. On the other hand, if Snowden were to claim that knowledge is both a thing that does not flow, on the one hand, and a thing that does flow on the other, then we would indeed have a contradiction or a paradox. But this does not seem to be what he is saying at all. Rather, what he seems to be saying is that knowledge flows - not that knowledge is flow, but that it (as a 'thing') is subject to movement. With this we agree. But where's the paradox in that?

Another possible interpretation of Snowden's claims about knowledge as flow is that he's really not talking about knowledge at all. Rather, he's talking about a process whose outcomes are knowledge (i.e., learning and innovation). But here we encounter, once again, the product/process confusion we covered before. The flow of knowledge (process) should not be regarded as knowledge. Both are things, but they are not the same things. The flow of knowledge occurs between various stages (or states) in the processes of knowledge production and integration, but to say that knowledge flows between the stages of a process is not to say that knowledge is a flow.

Turning to other sources for what flow could possibly mean to Snowden in this context, we see the term heavily used in two fields closely related to 
Knowledge Management. One is complex adaptive systems (CAS) theory, a bedrock of Snowden's own hypothesis, and the other is system dynamics, a closely related field in which the nonlinearity of complex systems is modeled and studied.

To CAS theorists, flows are movements of things between nodes and across connectors in networks (Holland, 1995, p. 23). In Holland's treatment of this subject, he states: "In CAS the flows through these networks vary over time; moreover nodes and connections can appear and disappear as the agents adapt or fail to adapt. Thus neither the flows nor the networks are fixed in time. They are patterns that reflect changing adaptations as time elapses and experience accumulates." (ibid.). Now, if this is what Snowden (and Stacey) mean by "ephemeral, active process[es] of relating," (Snowden, 2002, p. 101), again, we fail to see the paradox and see only confusion, instead. Holland and other CAS theorists are not claiming that the things that flow across ephemeral networks are the same things as the ephemeral networks, themselves. A sharp distinction between the two is made with no paradox involved, nor any need for one. And so we fail to see how the use of the term 'flows' in the literature on CASs could be used to support Snowden's claim of a paradox in the view of knowledge or the Cynefin model.

In the system dynamics arena, "stocks and flows" are central to the lingua franca of the field. Flows in system dynamics refer to streams of things (which are otherwise held in "stocks") moving at different rates of speed and with different degrees of frequency, with or without delays. But flows as things are never confused with the things that they carry. And so here again, we fail to see how the historical use of the term 'flows' necessarily leads to any sort of contradiction or paradox.

In sum, while Snowden purports to use the term 'flow' as a noun (as in, knowledge is flow) in his definition of knowledge, his actual use of the term in his discussion seems confined to its use as a verb (as in, knowledge flows). Thus, he never manages to provide a satisfactory definition for knowledge as flow. On the other hand, to the extent that he implies that flow may be a process, the process he refers to is arguably one that produces and/or transfers knowledge, but which is not the same as knowledge itself. For all of these reasons, we find Snowden's claim of a paradox in the third age definition of knowledge to be unpersuasive and full of confusions.

The Model: What Snowden's Cynefin model seems to be most fundamentally about is the dynamics of knowledge production and transfer in organizations. As discussed immediately above, this seems to be the thrust of his use of the term flows, although in most cases he seems to be talking more about transfer than production, an understanding which is 
encouraged by his graphical representation of flows across the boundaries contained in his model (See Figure 4 in Snowden, 2002, p. 9). This seems clearly intended to depict the flow of knowledge (things) from one Cynefin domain to another.

In further support of this view, many references in Snowden's account of the flows within and between his four domains can be found in his description of the model, including the following statement:

"In the third generation, we create ecologies in which the informal communities of the complex domain can self-organize and self manage their knowledge in such a way as to permit that knowledge to transfer to the formal, knowable domain on a JIT [just-in-time] basis." (ibid. p. 108).

Elsewhere, he makes similar repeated references to identifying and codifying knowledge, conveying it, transferring it, communicating it, and sharing it. Separately, he refers to knowledge creation, sense-making, pattern forming, learning, and innovation - all presumably references to knowledge production, not knowledge sharing or transfer.

Commentary: When viewed from the generational view of KM (McElroy, 1999, 2002), Snowden's emphasis on knowledge flows within and across the Cynefin model (sharing and transfer) seems decidedly supply-side in its orientation. Separately, his lesser emphasis on knowledge production would seem to be demand-side in focus. If this is true, what Snowden is attempting to say is that knowledge production and integration are both social processes which occur in different organizational settings, or ecologies, the awareness of which by managers should trigger different styles of interventions and oversight to cope with their effects. But if this is the case, what's the difference between Snowden's account of the coming third age of $\mathrm{KM}$ and the second generation of $\mathrm{KM}$ that was first identified (McElroy, 1999) and articulated four years ago? Further, from a second generation KM point of view, why should we view the Cynefin model as anything other than a personal and parochial depiction (or theory) of knowledge processing that can easily be accommodated within the existing framework of the second generation, KLC framework?

Indeed, Snowden's implicit claim that people in organizations tend to selforganize around the production and integration of knowledge is part and parcel of the KLC framework first articulated four years ago - as was the intentional and careful application of CAS theory to KM. Similarly, the view that behavioral patterns in knowledge processing form as a consequence of such self-organizations, and that they tend to oscillate between order, chaos, and complexity was also explicitly embraced in the second generation view of KM developed four years ago. And finally, the notion of 
choosing management interventions on the basis of awareness of all of this is an idea that first appeared in 1999 (McElroy, ICM Speech, April 1999a), and which later led to at least one formally defined method (McElroy and Cavaleri patent, The Policy Synchronization Method, 2000).

So even the idea of crafting management policies with the intention of synchronizing them with the self-organizing patterns of social knowledge processing behaviors in organizations is at least three years old, and is very much a part of existing, second generation thinking. On the basis of all of this, then, we continue to see no compelling reason to accept the claim that a new age in $\mathrm{KM}$ is upon us. What is upon us, perhaps, is a new model, formulated in a highly questionable and confusing fashion, that fits within the conceptual framework of second generation thinking, but not a new conceptual framework that would suggest the arrival of a new generation, stage, or age.

\section{Cynefin Conclusions}

The Cynefin model is an elaborate construct full of implications and hypotheses, but it is (1) also full of many difficulties and confusions, and (2) as presented by Snowden, it does not provide the conceptual framework one needs to compare his coming third age of KM to the first two. We will consider this second conclusion in the next section in a more general context. Here we note the many questions we raised about the Cynefin model in almost every detail. In our view, the model should only survive if its foundations are formulated much more rigorously and systematically.

- The reduction of the concept of cultural variation to the teaching/learning dichotomy should be abandoned even if it costs Snowden his four-category classification of common sense-making environments. Simplicity and ease of exposition to executives desperately trying to understand knowledge management must give way to reality in modeling sense-making environments.

- The concept of abstraction needs to be clarified so its meaning is clear to readers.

- If there are more than four types that can be composed out of the Cynefin fundamental attributes (as is indicated by our questions about alternative sense-making environments than may have been specified), they should be presented by Snowden. A classification framework must be evaluated as a whole, so that we can better understand the principles behind it. It should not be presented by describing only the categories its author thinks are important, because the unimportant categories may contain important insights that either reinforce or call into question the whole framework. Snowden's mention of, and 
decision to overlook, his fifth domain in the middle of the Cynefin model is unfortunate in this regard.

- The number of systems used to describe sense-making environments should increase. The existence of PCASs suggests that Snowden's system classification is incomplete. But even if one declined to explore the "mapping" of system types on to sense-making environments, it is very clear that Snowden's four types exhaust only a fraction of the logical possibilities suggested by his underlying concepts, and he provides no reasons for restricting Cynefin to his initial selection of four types

- The confusion between subject and object in talking about sense-making environments should be clarified and the one-toone mapping of system to Cynefin categories should be abandoned.

- The many small questions we have raised above on issues such as how leadership correlates to the different types should be answered.

- The concept of 'knowledge as flow' needs to be clarified so its meaning is clear to readers, especially the sense in which its meaning supposedly leads to the paradox claimed by Snowden. Short of that, the paradox claim should be abandoned.

- The degree to which the Cynefin model constitutes a material or conceptual departure or evolution from the currently existing (and previously developed) articulation of second generation $\mathrm{KM}$, if at all, should be demonstrated. Short of that, the Cynefin model should be seen as nothing more than a particular, and evidently, highly questionable, expression of second generation thinking, the essence of which has already been widely articulated. (See, for example: Albors G, 2001; Allee, 1997; Kelly and Allison, 1999; Bennet and Bennet, 2000, 2001; Carrillo, 1998, 2001; Cavaleri and Reed, 2000, and 2001; Courtney, Chae, and Hall, 2000; Firestone, 1998, 1999, 1999a, 1999b, 1999c, 2000, 2000a, 2000b, 2001, 20001a, 2001b, 2002; Kuscu, 2001; Loverde, 2001; McElroy, 1999a, 1999b, 1999c, 2000a, 2000b, 2000c, 2001, 2002, 2002a,; McMaster, 1996; Murray, 2000).

Even if all of the above points were met and the Cynefin model was made more multi-dimensional, it could still not serve as the basis for a new generation of knowledge management. The reason for this is that Cynefin is about sense-making and decision making; it is not a general conceptual framework that can function as an intellectual umbrella for all activities in the field of KM. Instead, it illuminates one corner of the concerns of KM, the corner that deals with the foundations of (and immediate precursors to) action. It is an important corner, even a fundamental one. But it does not 
provide a framework for approaching knowledge production and integration, or the role of knowledge claim evaluation in knowledge production, or knowledge management, or KM-related metrics, or sustainable innovation, or a comprehensive information technology system supporting $\mathrm{KM}$, or $\mathrm{KM}$ software evaluation, or intellectual capital, or the type of enterprise that will support sustainable innovation, or many other subjects that are important for the emergent discipline that is KM.

\section{Conclusion: The Three Stages, The Three Ages, the Two Generations and Comparative Frameworks}

Perhaps the most important differentiator between the three views of change in KM we have analyzed here is the methodology used to analyze change in the three instances. Basically, Koenig and Snowden take a story-telling approach to analyzing changes in the $\mathrm{KM}$ evolutionary process, whereas McElroy bases his case for fundamental change on the KLC knowledge processing framework and the distinction between knowledge processing and $\mathrm{KM}$.

Koenig takes an IT approach to KM and basically tells a story of changes in IT-related concerns. Thus, he starts by noting that the first stage of KM was about using the Internet for knowledge sharing and transfer. The second stage was a reaction to the failure of the first to live up to its promise by failing to take account of human factors essential to make IT applications successful, and the third stage is about improving the IT side by making it easier for humans to navigate to the information or knowledge they want or need.

This story of changes occurring in response to a desire to make IT-based KM solutions successful does not specify a conceptual framework based on concepts of knowledge, KM, business processing and outcomes. Lacking such a framework, Koenig has no tool to compare the three stages of $\mathrm{KM}$ in order to evaluate the comprehensiveness of change in its key elements. That is why his analysis seems ad hoc and questionable from the standpoint of whether the changes he records are really so fundamental as to suggest new stages in the KM evolutionary process.

The situation is little better with Snowden's approach. Boiled down to its essentials, he almost seems to be saying:

- The first age was about applying the BPR notions of Hammer and Champy (1993) on a foundation of Taylor (1912);

- The second age was about applying the vision expressed in Nonaka and Takeuchi (1995); and

- The coming third age will be about applying the vision expressed in his own Cynefin model, coupled with Stacey's 
notions about the paradoxical character of knowledge, and expanded through its synthesis with the systems typology.

So, Snowden's story of change is not guided by a transcendent conceptual framework that can provide us with categories to set a context for describing change, but rather is a claim that $\mathrm{KM}$ proceeds from vision to vision expressed in great books and/or articles. His view provides no guide about what the next fundamental change in KM will bring, because how can we know what the rest of a story might be?

McElroy's (1999) approach to change uses the conceptual framework of the Knowledge Life Cycle (KLC) to analyze the change in KM that he believes suggests there have been two and only two generations so far. The KLC framework clearly distinguishes knowledge production and knowledge integration processes as the two processes comprising knowledge processing behavior. In turn, these fundamental processes are divided into four sub-processes for each process. Figure 1 above provides enough detail to allow one to recognize that knowledge processing activities are clustered in either the knowledge production or knowledge integration categories, and that $\mathrm{KM}$ initiatives have also primarily been concerned with either one or the other. Once that recognition was made, it was easy to see that the early period of formal KM, from the early 90 s to at least 1999, has primarily been about knowledge integration, and that SGKM, the fusion of concern about knowledge integration with knowledge production, begins only in the late '90s and is first explicitly formulated against the backdrop of the Knowledge Management Consortium International KMCl, including the authors' prior works: Firestone (1998, 1999, 1999a, 2000) and in McElroy's (1999) article.

The SGKM "paradigm" of fusion between supply- and demand-side KM now exists alongside the continuing practice of supply-side $\mathrm{KM}$, which is still dominant in the field. But the growing concern with innovation in corporate, government, and intellectual capital circles suggests that further fundamental change in $\mathrm{KM}$ is unlikely until there is a much wider embrace of demand-side problems. If, however, fundamental change were to occur, the KLC framework suggests that it will revolve around a reconceptualization of knowledge processing, involving a specification of some new fundamental process in addition to knowledge production and integration, or perhaps a fundamental re-conceptualization of knowledge production or knowledge integration processes. The fact that neither the Koenig nor Snowden views of change focus on such an evolution in how we see knowledge processing explains why the changes they focus on do not add up to a new stage, age, or generation of KM. 


\section{References}

Albors G., J. (2001), "Knowledge Creation in an SME Environment", Knowledge and Innovation: Journal of the KMCl, 1, no. 2, 145-160.

Allee, V. (1997). The Knowledge Evolution: Expanding Organizational Intelligence, Boston, MA: Butterworth-Heinemann.

Amidon, D. (1997), Innovative Strategy for the Knowledge Economy: The Ken Awakening, Boston, MA: Butterworth Heinemann.

Bennet, A and Bennet, D. (2001) "Exploring Relationships in the Next Generation Knowledge Organization" Knowledge and Innovation: Journal of the KMCl, 1, no.2, 91- 109.

Bennet, A and Bennet, D. (2000) "Characterizing the Next Generation Knowledge Organization" Knowledge and Innovation: Journal of the $\mathrm{KMCl}$, 1, no.1, 8-42.

Birch, D. and Veroff, J. (1966), Motivation: A Study of Action, Belmont, CA: Brooks/Cole.

Boisot, M. (1998), Knowledge Assets, Oxford University Press.

Brown, J. S. (1995), "The People Are the Company," Fast Company, 1.

Brown, J. S. and Duguid, P. (2000) The Social Life of Information, Cambridge, MA: Harvard Business School Press.

Brown, J. and Duguid, P. (1991), "Organisational Learning And Communities Of Practice," Organisation Science, March, 40-57.

Carrillo, F. J. (1998), "Managing Knowledge-based value Systems", Journal of Knowledge Management, 1, No. 4, (June), 280-286.

Carrillo, F. J. (2001), "Meta-KM: A Program and a Plea", Knowledge and Innovation: Journal of the $\mathrm{KMCl}, \mathbf{1}$, no. 2, 27-54.

Cavaleri, S. and Reed, F. (2000), "Designing Knowledge Generating Processes", Knowledge and Innovation: Journal of the KMCl, 1, no. 1, 109131.

Cavaleri, S. and Reed, F. (2001), "Organizational Inquiry: The Search for Effective Knowledge," Knowledge and Innovation: Journal of the $K M C l, 1$, no. 3, 27-54. 
Courtney, J., Chae, B. and Hall, D. (2002) "Developing Inquiring Organizations Knowledge and Innovation: Journal of the $K M C l, 1$, no. 1, 132-145.

Davenport, T. and Prusak, L. (1997), Working Knowledge: How Organizations Manage What They Know, Boston, MA: Harvard Business School Press.

Denning, S. (2001), The Springboard, How Storytelling Ignites Action in Knowledge-era Organizations, Boston, MA: KMCl Press/Butterworth Heinemann.

Denning, S. et.al. (1998), What is Knowledge Management? Washington, DC: World Bank.

Firestone, J. M. (2002), Enterprise Information Portals and Knowledge Management, Boston, MA: KMCI Press/Butterworth Heinemann.

Firestone, J. M. (2001), "Key Issues in Knowledge Management", Knowledge and Innovation: Journal of the $\mathrm{KMCl}, 1$, no. 3, 8-38. Available at: http://www.dkms.com/White Papers.htm.

Firestone, J. M. (2001a), "Knowledge Management Process Methodology", Knowledge and Innovation: Journal of the KMCl, 1, no. 2, 85-108. Available at: http://www.dkms.com/White Papers.htm.

Firestone, J. M. (2001b) "Enterprise Knowledge Portals, Knowledge Processing and Knowledge Management," in Ramon Barquin, Alex Bennet, and Shereen Remez, (eds.) Building Knowledge Management Environments for Electronic Government, Vienna, VA: Management Concepts.

Firestone, J. M. (2000). "Accelerated Innovation and KM Impact," Financial Knowledge Management, (Q1, 2000), 54-60.

Firestone, J. M. (2000a) "Knowledge Management: A Framework for Analysis and Measurement," White Paper No. 17, Executive Information Systems, Inc., Wilmington, DE, October 1, 2000, Available at: http://www.dkms.com/White_Papers.htm.

Firestone, J. M. (2000b), "Enterprise Knowledge Portals: What They Are and What They Do," Knowledge and Innovation: Journal of the KMCl, 1, no. 1, 85-108. Available at: http://www.dkms.com/White Papers.htm. 
Firestone, J. M. (2000c), "Enterprise Knowledge Portals and e-Business Solutions," White Paper No. 16, Executive Information Systems, Inc., Wilmington, DE, October 1, 2000, Available at: http://www.dkms.com/White_Papers.htm.

Firestone, J. M. (1999). "Enterprise Knowledge Management Modeling and Distributed Knowledge Management Systems," Executive Information Systems White Paper, Wilmington, DE, January 3, 1999. Available at http://www.dkms.com/White Papers.htm.

Firestone, J. M. (1999a), " The Artificial Knowledge Manager Standard: A Strawman," Executive Information Systems KMCI Working Paper Paper No. 1. Wilmington, DE Available at http://www.dkms.com/White Papers.htm

Firestone, J. M. (1999b). "Enterprise information Portals and Enterprise Knowledge Portals," DKMS Brief, 8, Executive Information Systems, Inc., Wilmington, DE, March 20, 1999

Firestone, J. M. (1999c) "The Metaprise, the AKMS, and the Enterprise Knowledge Portal," Working Paper No. 3, Executive Information Systems, Inc., Wilmington, DE, May 5, 1999, Available at: http://www.dkms.com/White_Papers.htm.

Firestone, J. M. (1998) "Knowledge Management Metrics Development: A Technical Approach," Executive Information Systems White Paper, Wilmington, DE, June 25, 1998. Available at http://www.dkms.com/White Papers.htm.

Firestone, J. M. and McElroy, M. W. (2002), Certified Knowledge and Innovation Manager (CKIM) Level I Course Notes (section on the Open Enterprise available from the authors).

Haeckel, S. H., (1999). Adaptive Enterprise, Boston, MA: Harvard Business School Press.

Hammer, M. and Champy, J. (1993), Re-engineering the Corporation, New York, NY: Harper Business.

Holland, J. H. (1995). Hidden Order Reading, MA: Addison-Wesley.

Keesing, R. and Strathern, A. (1997), Cultural Anthropology: A Contemporary Perspective, (3 $3^{\text {rd }}$ edition) Belmont, CA: Wadsworth.

Kelly S. and Allison, M. A. The Complexity Advantage (New York, NY: Business Week Books/McGraw-Hill, 1999). 
Koenig, Michael E. D. (2002), "The third stage of KM emerges," KMWorld 11, No. 3 (March, 2002) 20-21, 28.

Kuhn, T. (1970), The Structure of Scientific Revolutions, Chicago, IL: University of Chicago Press, (1970 edition).

Kuscu, I. (2001), "An Adaptive Approach to Organisational Knowledge Management", Knowledge and Innovation: Journal of the $K M C l, 1$, no. 2, 110-127.

Leonard-Barton, D. (1995), Wellsprings of Knowledge, Boston, Harvard Business School Press.

Loverde, L. (2001), "Intellectual Capital: An M \& A Approach "Knowledge and Innovation: Journal of the $K M C l, 1$, no. 3, 58-88.

McElroy, M. W. (2003). The New Knowledge Management: Complexity, Learning, and Sustainable Innovation, Boston, MA: KMCI

Press/Butterworth-Heinemann.

McElroy, M. W. (2002), "Social Innovation Capital," Journal of Intellectual Capital (Vol. 3, No. 1, 2002), pp. 30-39.

McElroy, M. W. (2002a), "A Framework For Knowledge Management," Cutter IT Journal (March 2002, Vol. 15, No. 3), pp. 12-17.

McElroy, M. W. (2001) "Where Does KM Belong? A Better Solution," Knowmap: The Knowledge Management, Auditing and Mapping Magazine (Vol. 1, No. 4, 2001) (www.knowmap.com)

McElroy, M. W. (2000), "Using Knowledge Management To Sustain Innovation," Knowledge Management Review (Sept./Oct. 2000, Vol. 3, Issue 4), pp. 34-37.

McElroy, M. W. (2000a), "Integrating Complexity Theory, Knowledge Management, and Organizational Learning," Journal of Knowledge Management Vol. 4 No. 3 (2000), pp. 195-203.

McElroy, M. W. (2000b), "The New Knowledge Management," Knowledge And Innovation: Journal of the $\mathrm{KMCl}$ (October 15, 2000, Vol. 1, No. 1), pp. 43-67.

McElroy, M. W. and Cavaleri, S. A. (2000c), "Policy Synchronization Method", Patent application filed with the U.S. Patent and Trademark Office in September 2000. 
McElroy, M. W. (1999). "The Second Generation of KM," Knowledge Management (October 1999) pp. 86-88.

McElroy, M. W. (1999a). ICM Speech in Miami, FL, April 1999: "The Knowledge Life Cycle, An Executable Model For The Enterprise." Available at http://www.macroinnovation.com/images/KnlgLifeCycle.pdf

McElroy, M. W. (1999b). "Double-Loop Knowledge Management," Systems Thinker (October 1999, Vol. 10, No. 8) pp. 1-5.

McElroy, M. W. and Cavaleri, S. A.: Policy Synchronization Method: Patent application filed with the U.S. Patent and Trademark Office in September 2000.

McMaster, Michael D. (1996). The Intelligence Advantage: Organizing For Complexity, Boston, MA: Butterworth-Heinemann.

Murray, A. J. (2000). "Knowledge Systems Research," Knowledge and Innovation: Journal of the KMCl, 1, no. 1, 68-84.

Nonaka, I. and Takeuchi, H. (1995), The Knowledge Creating Company, New York, NY: Oxford University Press.

Nonaka, I (1991), "The Knowledge-Creating Company," Harvard Business Review, November-December.

Polanyi, M. (1958). Personal Knowledge, Chicago, IL: University of Chicago Press, 1958.

Polanyi, M. (1966). The Tacit Dimension, London, UK: Routledge and Kegan Paul.

Rumizen, M. (1998), "How Buckman Laboratories 'Shared Knowledge' Sparked a Chain Reaction", The Journal for Quality \& Participation, 1, 3438.

Senge, P. The Fifth Discipline: The Art \& Practice of the Learning Organization, New York, NY: Currency Doubleday, 1990.

Smythe, E. (1997) "Life in Organizations: Sense-making or Appreciation? A Comparison of the Works of Karl Weick and Geoffrey Vickers," "unpublished draft", available at: http://mis.commerce.ubc.ca/smythe/weick.pdf. 
Snowden, D. (2002) "Complex Acts of Knowing; Paradox and Descriptive Self-awareness", Journal of Knowledge Management, 6, no. 2 (May) 1-14.

Snowden, D. (2000), "Cynefin: a sense of time and space, the social ecology of knowledge management", in Knowledge Horizons : The Present and the Promise of Knowledge Management ed. C Despres \& D Chauvel, Butterworth Heinemann October 2000.

Snowden, D. (1999), "Liberating Knowledge" Introductory chapter to Liberating Knowledge CBI Business Guide, Caspian Publishing October 1999

Stacey, R. D. (2001). Complex Responsive Processes in Organizations: Learning and Knowledge Creation, New York, NY: Routledge.

Stacey, R. D. (1996). Complexity and Creativity in Organizations, San Francisco, CA: Berrett-Koehler.

Stewart, Thomas (1999), Intellectual Capital: The New Wealth of Organizations, New York, NY: Currency Doubleday.

Taylor, F. (1912), Testimony at Congressional hearing, January 25, 1912.

Weick, K. (1995), Sense-making In Organisations, Beverly Hills, CA: Sage Publications.

Wenger, E. (1999) "Communities of Practice: The Key to Knowledge Strategy," Knowledge Directions: The Journal of the Institute For Knowledge Management $t_{1}$ 48-63.

Wheatley, M. and Kellner-Rogers, M. (1996), A Simpler Way, San Francisco, CA: Berrett-Koehler Publishers.

Wiig, K., (1995) Knowledge Management Methods: Practical Approaches to Managing Knowledge, Arlington, TX: Schema Press.

Wiig, K., (1994) Knowledge Management: The Central Focus for Intelligent Acting Organization, Arlington, TX: Schema Press.

Wiig, K., (1993) Knowledge Management Foundations - Thinking about Thinking - How People and Organizations Create, Represent, and Use Knowledge, Arlington, TX: Schema Press.

Wiig, K. (1989), Managing Knowledge: A Survey of Executive Perspectives, Arlington, TX: Schema Press. 


\section{Biographies}

\section{Joseph M. Firestone, Ph.D.}

Joseph M. Firestone, Ph.D. is Vice-President and Chief Knowledge Officer (CKO) of Executive Information Systems (EIS), Inc. Joe has varied experience in consulting, management, information technology, decision support, and social systems analysis. Currently, he focuses on product, methodology, architecture, and solutions development in Enterprise Information and knowledge Portals, where he performs Knowledge and knowledge management audits, training, and facilitative systems planning, requirements capture, analysis, and design. Joe was the first to define and specify the Enterprise Knowledge Portal (EKP) Concept, and is the leading writer, designer, commentator, and trainer in this area. He is widely published in the areas of Decision Support (especially Enterprise Information and Knowledge Portals, Data Warehouses/Data Marts, and Data Mining), and Knowledge Management, and has completed a fulllength industry report entitled "Approaching Enterprise Information Portals." $\mathrm{He}$ is also the author of Enterprise Information Portals and Knowledge Management. (Boston, $\mathrm{MA}: \mathrm{KMCl}$ Press/Butterworth-Heinemann, forthcoming, 2002)

Joe is a founding member of the Knowledge Management Consortium International $(\mathrm{KMCl})$, Its Corporate Secretary, and Executive Vice President of Education, Research, and Membership, the CEO in these areas, directly responsible to KMCl's Board. $\mathrm{He}$ is also the Director of the $\mathrm{KMCl}$ Knowledge and Innovation Manager Certification (CKIM) Program (See, http://www.kmci.org/Institute/certification/ckim_details.htm), and, Director of the $\mathrm{KMCl}$ Research Center, and Editor of the new journal Knowledge and Innovation: Journal of the $\mathrm{KMCl}$. Joe is also a frequent speaker at national conferences on KM and Portals, and a trainer in the areas of Enterprise Information Portals, Enterprise Knowledge Portals and Knowledge Management (KM). He is also developer of the web site www.dkms.com, one of the most widely visited web sites in the Portal and KM fields. DKMS.COM has now reached a visitation rate of 135,200 visits and an access ("hit") rate of 1,014,000, both annually.

Joe can be reached at eisai@comcast.net, or by phone at 703-461-8823

\section{Mark W. McElroy}

Mark W. McElroy is president and CEO of Macroinnovation Associates, LLC, a knowledge and innovation management consultancy based in 
Windsor, VT. Mark is also president of the Knowledge Management Consortium International (KMCl). $\mathrm{He}$ is a twenty-five year veteran of management consulting, having spent much of that time at Price Waterhouse, and later as a partner at KPMG. More recently, Mark was a principal in IBM's Knowledge Management Consulting practice in Cambridge, MA. In 2000, he formed his own consultancy and went on to develop the Policy Synchronization Method, an innovation management method based on complexity theory that currently holds patent-pending status in the U.S.

Mark is also one of the chief architects of 'the new knowledge management,' a perspective on KM that sees innovation as a selforganizing social process that can be measured and valued as such. $\mathrm{He}$ has just completed a new book by the same name, The New Knowledge Management - Complexity, Learning, and Sustainable Innovation (Butterworth-Heinemann, 2003), which will be released in the fall of this year (2002).

Mark can be reached at mmcelroy@vermontel.net. His company's website can be found at www.macroinnovation.com. 